December 2010 - WUB/10-35

(v3) December 2011

\title{
Supersymmetric Higgs production in gluon fusion
}

\author{
Robert V. Harlander, Franziska Hofmann, Hendrik Mantler \\ Fachbereich $C$, Bergische Universität Wuppertal \\ 42097 Wuppertal, Germany
}

\begin{abstract}
The cross section through gluon fusion is calculated for the production of the light neutral Higgs boson through next-to-leading order QCD within the Minimal Supersymmetric Standard Model. The quark-mediated contributions are taken into account exactly, while for the genuinely supersymmetric terms we use expressions obtained in the limit of large squark, gluino and top quark masses. We present numerical results for the total inclusive cross section as well as for kinematical distributions of the Higgs boson. We also consider the effect of an MSSM-like $4^{\text {th }}$ generation on the total Higgs production cross section.
\end{abstract}

\section{Introduction}

With the first sensitivity of the Tevatron to a Higgs signal [1] 1 and the first data taken at the Large Hadron Collider (LHC), Higgs phenomenology has received an additional significant boost in the last few years. It is pleasing to see that the efforts towards precise predictions for Higgs production (for reviews, see Refs. [2 [5]) at the LHC are paying off, currently leading to significant bounds on the Standard Model (SM) Higgs boson mass.

Another interesting application of the on-going Higgs searches are the restrictions on parameters of theories that go beyond the SM, such as the Minimal Supersymmetric Standard Model (MSSM) [6] (see also Ref. [7]) or the SM with a fourth fermion generation [8. ${ }^{1}$

One of the most important production mechanisms for these searches is gluon fusion which is known in the SM through next-to-next-to-leading order (NNLO) QCD [9 19. Effects beyond that order have been investigated as well [20 25], with the reassuring conclusion that the theory uncertainty derived from the renormalization and factorization scale variation of the fixed order NNLO result seems to be reliable. Electro-weak (EW) corrections have been found to be below around $6 \%$ relative to the leading order (LO)

\footnotetext{
${ }^{1}$ See http://tevnphwg.fnal.gov/ for updates.
} 
result [26, 27], and a calculation of the mixed QCD/EW effects in the unphysical limit $M_{h} \ll M_{W}$ supports the assumption of a factorization of QCD and EW corrections [28].

Concerning the MSSM, at LO squark loop effects have to be taken into account. Due to supersymmetry (SUSY), their couplings to the Higgs bosons are typically suppressed by powers of $M_{q} / \tilde{M}_{q}$, which is why they only contribute significantly to the cross section if the squark mass $\tilde{M}_{q}$ is not too large. In that case, however, there are certain regions of the SUSY parameter space where a quite drastic cancellation among the quark and the squark induced amplitudes may occur [29,30].

Apart from the SM-like diagrams involving only quarks and gluons, QCD corrections to gluon fusion in the MSSM require the calculation of the corresponding corrections to the LO squark diagrams, plus diagrams involving quarks, squarks, and gluinos simultaneously. Meanwhile, all the ingredients for the next-to-leading order (NLO) QCD corrections to gluon fusion in the MSSM have been calculated: The top/stop/gluino effects were taken into account through an effective Lagrangian approach [31 34, a result for scalar QCD (scalar quarks with arbitrary mass) was calculated both numerically and analytically [3537, and for the bottom/sbottom/gluino diagrams recently a result in terms of an expansion in the inverse SUSY masses was presented [38]. The quark/squark/gluino effects have also been calculated fully numerically both for the top and the bottom sector [39]. Very recently, even the NNLO effects in the heavy top/stop limit have been evaluated [40].

In this paper, we present a calculation of the full NLO QCD effects for hadronic Higgs production in the MSSM with real parameters and combine it with the NNLO QCD effects from purely top-quark induced contributions known from the SM calculations. Although most of our results should be valid for Higgs masses up to $M_{h} \lesssim 2 \min \left(M_{t}, \tilde{M}\right)(\tilde{M}$ is the typical scale of the SUSY partner masses), we present results only for the phenomenologically most relevant case of the light MSSM Higgs boson, for which $M_{h} \lesssim 135 \mathrm{GeV}$ (see, e.g. Refs. [41, 42] for reviews, and Ref. [43] for the most up-to-date result). We have re-calculated all of the real corrections due to quarks and squarks, while for the virtual corrections to the pure quark terms the analytic result of Ref. [44] is employed. These results are valid for general quark/squark masses and can therefore be used for the top as well as the bottom sector. Concerning the virtual squark effects, we use the effective Lagrangian approach of Ref. 32 for the top sector, evaluating the Wilson coefficient with the help of evalcsusy.f [45]. For the bottom sector, we follow the approach of Ref. [38,46] in order to evaluate pure sbottom and the bottom/sbottom/gluino diagrams in terms for $1 / \tilde{M}_{b}$, where $\tilde{M}_{b}$ denotes the average sbottom and gluino mass.

Apart from the inclusive total cross section, we also present kinematical distributions for the Higgs boson: while the transverse momentum $\left(p_{T}\right)$ and pseudo-rapidity $(\eta)$ distributions at $\mathcal{O}\left(\alpha_{s}^{3}\right)$ have to be considered as LO (at $\mathcal{O}\left(\alpha_{s}^{2}\right)$, it is $p_{T} \equiv 0$ and $\eta=\infty$ ) and have been evaluated before [47-49], to our knowledge this is the first time that rapidity 
(y) distributions are being presented in the full MSSM at this order. Our calculations are based on a numerical routine which will be made publicly available and can be obtained from the authors upon request.

The remainder of this paper is organized as follows: Section 2 outlines our notation, describes our calculation and methods, and quotes some of the most important formulas. Section 3 defines the scenarios for our numerical analyses, describes how we obtain our best prediction of the cross section, and provides numerical results for the total inclusive cross section. Section 4 presents results for the LO transverse momentum and the NLO rapidity distribution of the Higgs boson. Section 5 considers the effect of an MSSM-like $4^{\text {th }}$ generation on the total inclusive cross section through NLO QCD, and Section 6 contains our conclusions. In the Appendix, we collect the Feynman rules for the Higgs couplings used in this paper, give some more analytical formulas, and provide numerical results for other SUSY scenarios.

\section{Calculation of the cross section}

\section{$2.1 \quad$ General outline}

For the sake of clarity, we collect some of the most important notation at this point. Concerning the SUSY parameters, we follow the usual notation (as mentioned before, we work in the MSSM with real parameters):

- The angle $\alpha$ rotates the CP even neutral components of the two Higgs doublets $H_{1}$ and $H_{2}$ into their mass eigenstates $h, H$, where by definition $M_{h}<M_{H}$. In all our formulas, $\alpha$ actually denotes the loop-corrected effective mixing angle $\alpha_{\text {eff }}$.

- The ratio of the two Higgs vacuum expectation values is denoted by $\tan \beta=v_{2} / v_{1}$.

- The coefficient of the bilinear term $\sim H_{1} \cdot H_{2}$ in the superpotential is called $\mu_{\text {SUSY }}$. At tree-level, it is related to other SUSY parameters as in Eq. (36) of Appendix A.

Furthermore, the following abbreviations will be applied:

$$
\begin{array}{ll}
l_{q s}=\ln \frac{M_{q}^{2}}{\tilde{M}_{q}^{2}}, & l_{r q}=\ln \frac{\mu_{\mathrm{r}}^{2}}{M_{q}^{2}}, \quad L_{h q}=\ln \frac{M_{h}^{2}}{M_{q}^{2}}-i \pi, \\
\tau_{q}=\frac{4 M_{q}^{2}}{M_{h}^{2}}, \quad \tilde{\tau}_{q, i}=\frac{4 \tilde{M}_{q i}^{2}}{M_{h}^{2}},
\end{array}
$$

where $M_{q}$ and $\tilde{M}_{q i}$ denote the on-shell quark and squark mass $(q \in\{b, t\})$, while $\tilde{M}_{q}=$ $\left(\tilde{M}_{q 1}+\tilde{M}_{q 2}+\tilde{M}_{g}\right) / 3$ is the average of the $q$-squark and gluino $\left(\tilde{M}_{g}\right)$ masses. The mass 
of the light CP-even Higgs boson is called $M_{h}$, and $\mu_{\mathrm{r}}$ is the renormalization scale. The factorization scale will be denoted by $\mu_{\mathrm{f}}$.

Unless stated otherwise, the strong coupling constant will always be defined in the framework of standard five-flavor QCD, renormalized in the $\overline{\mathrm{MS}}$ scheme:

$$
\alpha_{s} \equiv \alpha_{s}^{(5), \overline{\mathrm{MS}}} .
$$

Following the notation of Ref. [12], we write the hadronic cross section at the hadronic center-of-mass energy $s$ through NLO QCD as

$$
\sigma(p p \rightarrow H+X)=\sigma_{0}\left[1+C \frac{\alpha_{s}}{\pi}\right] \tau_{h} \frac{\mathrm{d} \mathcal{L}^{g g}}{\mathrm{~d} \tau_{h}}+\Delta \sigma_{g g}+\Delta \sigma_{g q}+\Delta \sigma_{q \bar{q}},
$$

where

$$
\frac{\mathrm{d} \mathcal{L}^{g g}}{\mathrm{~d} \tau}=\int_{\tau}^{1} \frac{\mathrm{d} x}{x} g(x) g(\tau / x),
$$

with the gluon density $g(x)$ and $\tau_{h}=M_{h}^{2} / s$. The normalization factor $\sigma_{0}$ determines the LO cross section. In the framework of the MSSM, we write it as

$$
\sigma_{0}=\frac{G_{\mathrm{F}} \alpha_{s}^{2}\left(\mu_{\mathrm{r}}^{2}\right)}{288 \sqrt{2} \pi}|\mathcal{A}|^{2}, \quad \mathcal{A}=\sum_{q \in\{t, b\}}\left(a_{q}^{(0)}+\tilde{a}_{q}^{(0)}\right),
$$

where

$$
\begin{gathered}
a_{q}^{(0)}=g_{q} \frac{3 \tau_{q}}{2}\left(1+\left(1-\tau_{q}\right) f\left(\tau_{q}\right)\right), \quad \tilde{a}_{q}^{(0)}=-\frac{3 \tau_{q}}{8} \sum_{i=1}^{2} g_{q, i i}^{h}\left(1-\tilde{\tau}_{q, i} f\left(\tilde{\tau}_{q, i}\right)\right), \\
f(\tau)= \begin{cases}\arcsin ^{2} \frac{1}{\sqrt{\tau}}, & \tau \geq 1, \\
-\frac{1}{4}\left(\log \frac{1+\sqrt{1-\tau}}{1-\sqrt{1-\tau}}-i \pi\right)^{2}, & \tau<1 .\end{cases}
\end{gathered}
$$

The $a^{(0)}\left(\tau_{q}\right)$ and the $\tilde{a}^{(0)}\left(\tilde{\tau}_{q, i}\right)$ terms are due to quark and squark diagrams, respectively, as the ones displayed in Fig. 1. It may be useful to quote the relevant limits of these functions:

$$
\begin{aligned}
& a_{q}^{(0)} \stackrel{\tau_{q} \gg 1}{=} g_{q}^{h}\left[1+\frac{7}{30 \tau_{q}}+\cdots\right], \quad \tilde{a}_{q}^{(0)} \stackrel{\tilde{\tau}_{q, i} \gg 1}{=} \frac{\tau_{q}}{8} \sum_{i=1}^{2} \frac{g_{q, i i}^{h}}{\tilde{\tau}_{q, i}}\left[1+\frac{8}{15 \tilde{\tau}_{q, i}}+\cdots\right], \\
& a_{q}^{(0)} \stackrel{\tau_{q} \ll 1}{=} \frac{3}{2} g_{q}^{h} \tau_{q}\left[1-\frac{L_{h q}^{2}}{4}+\frac{\tau_{q} L_{h q}}{4}\left(1+L_{h q}\right)+\cdots\right] .
\end{aligned}
$$




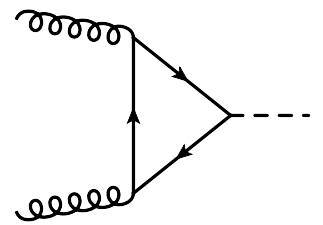

(a)

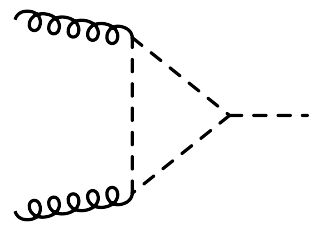

(b)

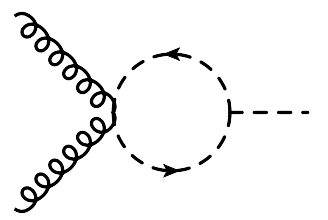

(c)

Figure 1: Feynman diagrams contributing to Higgs production in gluon fusion at LO.

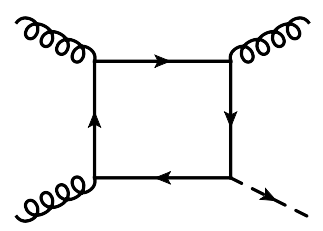

(a)

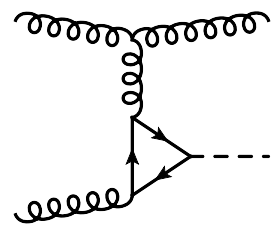

(b)

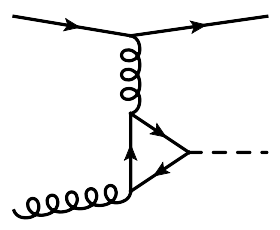

(c)

Figure 2: Sample Feynman diagrams contributing to the real corrections at NLO to Higgs production in gluon fusion. The particle in the loop can be a quark or a squark of bottom or top flavor.

The coupling constants $g_{q}^{h}$ and $g_{q, i j}^{h}$ are given in Appendix A those for $q=t$ can also be found in Ref. [32].

In principle, the sum in Eq. (5) should run over all quark flavors. However, only the top and the bottom quark account for a relevant contribution to the cross section, while all others are suppressed by their Yukawa coupling.

The quantities $\Delta \sigma_{g g}, \Delta \sigma_{g q}, \Delta \sigma_{q \bar{q}}$ in Eq. (3) denote the non-singular terms of the cross section arising from $g g, g q$ and $q \bar{q}$ scattering. Typical diagrams are shown in Fig. 2 , the $q \bar{q}$ contribution is obtained through crossing from Fig.2(c). They can be evaluated using wellknown techniques. We have expressed them in terms of Passarino-Veltman functions [50] and checked our result against the literature (see, e.g. Ref. [51/54]).

\section{$2.2 \quad$ Virtual corrections}

The coefficient $C$ in Eq. (3) denotes the virtual corrections to the $g g$ initiated process, regularized by the infrared singular part of the cross section for real gluon emission. We 


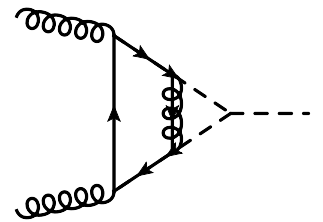

(a)

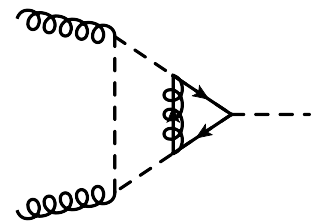

(b)

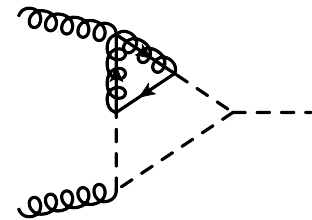

(c)

Figure 3: Sample diagrams for the mixed quark/squark/gluino contribution to gluon fusion at NLO.

write

$$
C=2 \operatorname{Re}\left[\mathcal{A}_{\infty}^{-1} \sum_{q}\left(a_{q}^{(1)}+\tilde{a}_{q}^{(1)}\right)\right]+\pi^{2}+\beta_{0} \ln \frac{\mu_{\mathrm{r}}^{2}}{\mu_{\mathrm{f}}^{2}},
$$

where $\beta_{0}=11 / 2-n_{l} / 3$ with $n_{l}=5$, and $\mathcal{A}_{\infty}$ is the LO amplitude in the limit of large stop and sbottom masses, i.e.,

$$
\mathcal{A}_{\infty}=\sum_{q}\left(a_{q}^{(0)}+\frac{\tau_{q}}{8} \sum_{i=1}^{2} \frac{g_{q, i i}^{h}}{\tilde{\tau}_{q, i}}\right) .
$$

The NLO quark-loop contribution $a_{q}^{(1)}$, corresponding to the SM part, has first been evaluated numerically a long time ago [12] and was later expressed in terms of analytic functions [35, 36, 44]. We provide a few terms of its small- and large-mass expansions in Appendix B,

One class of diagrams contributing to the SUSY part $\tilde{a}_{q}^{(1)}$ is obtained by attaching a virtual gluon to Fig.1(b) and (c). However, both squark and gluino effects need to be considered in order to preserve supersymmetry [31, 32]; sample diagrams containing a gluino are shown in Fig. 3. A fully numerical result for $\tilde{a}_{q}^{(1)}$ for general quark/squark/gluino masses was obtained in Ref. [39], but the corresponding code is not publicly available. For the pure squark diagrams, there exists both an analytic and a numerical result [35 37. In Ref. [31, 32, 34, $\tilde{a}_{q}^{(1)}$ was evaluated for the top sector (i.e., $q=t$ ) in terms of an effective Lagrangian, and one can write

$$
\tilde{a}_{t}^{(1)}=c_{1}^{(1)}-g_{t}^{h} \frac{11}{4}+\mathcal{O}\left(M_{h}^{2}\right),
$$

where $c_{1}^{(1)}$ is the NLO term of the Wilson coefficient, defined in Eq. (2.5) of Ref. [32. It can be evaluated with the help of the publicly available program evalcsusy. $f$ [45]. 
Concerning the SUSY bottom quark/squark sector, a fully numerical result for general masses was presented in Ref. [39]. Recently, this contribution was calculated with the help of asymptotic expansions in the limit of large SUSY masses [38. We present an independent result, following the same strategy. However, due to the SUSY mass spectrum emerging from the most popular SUSY breaking scenarios, we decided to take the limit $\tilde{M}_{b} \equiv \tilde{M}_{b 1}=\tilde{M}_{b 2}=\tilde{M}_{g}$ which leads to an extremely simple result ( $\tilde{M}_{g}$ is the gluino mass). Let us briefly describe the calculation.

For the pure sbottom diagrams (i.e., without gluinos), the procedure is very similar to the one applied in Ref. [17, 19, 55, 56, where top quark mass suppressed terms for the SM cross section $\sigma(g g \rightarrow H+X)$ were evaluated (mind you, through NNLO; here, we consider NLO only). The expansion of the mixed bottom/sbottom/gluino diagrams is a little more involved, but still rather straightforward due to the algorithmically defined method of asymptotic expansions (see, e.g. Ref. [57]). Let us consider an example:
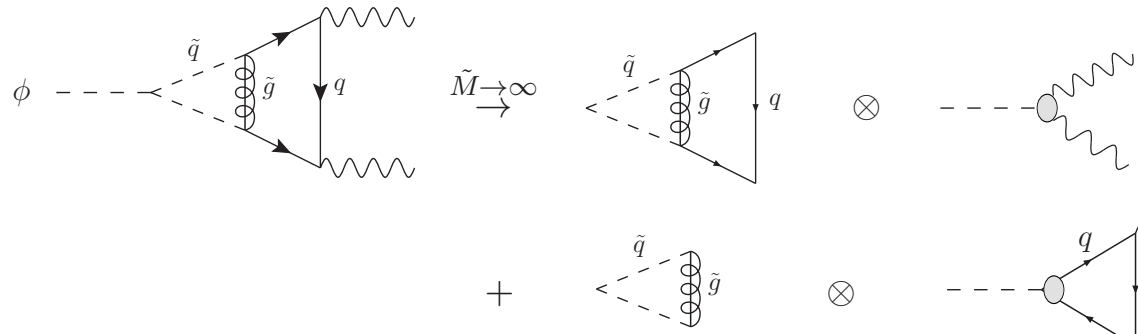

$\otimes$

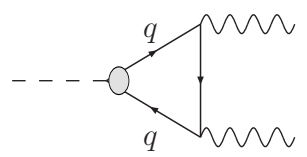

The notation is as follows: in the original diagram, Taylor-expand all the propagators of the sub-diagram $\gamma$ left of $\otimes$ in terms of $p / \tilde{M}$ before integration. Here, $p$ denotes any dimensional quantity (mass or momentum) of $\gamma$ except its loop momentum or $\tilde{M}$. The resulting Feynman integrals to evaluate in the above case are therefore: (i) one- and twoloop tadpole integrals (i.e., vanishing external momenta) depending on $\tilde{M}$, but not on $M_{b}$; (ii) one-loop vertex integrals with external momenta $q_{1}^{2}=q_{2}^{2}=0$ and $2 q_{1} \cdot q_{2}=M_{h}^{2}$, and mass $M_{b}$ (no dependence on $\tilde{M}$ ). Both types of integrals can be calculated analytically: type (i) with the help of MATAD [58], type (ii) by standard Passarino-Veltman reduction [50], for example. 
For clarity, let us consider another example:

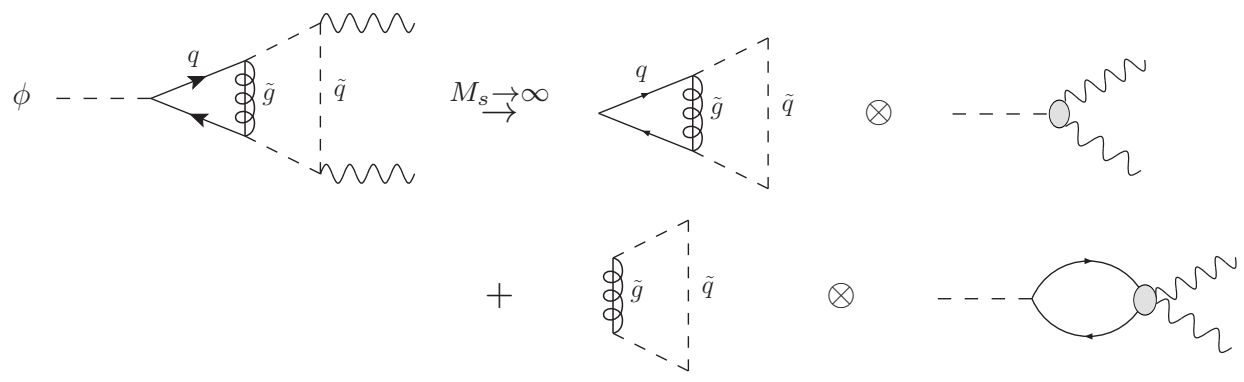

Using this procedure, the virtual corrections are valid for arbitrary values of $M_{h}$ and $M_{b}$, as long as they are both smaller than $22 \tilde{M}_{b}$.

We perform the calculation in Dimensional Reduction (DRED) by explicitely taking into account $\epsilon$-scalars as propagating particles. In order to avoid infra-red singularities in the corresponding Feynman integrals, we assign a large mass $M_{\epsilon}$ to the $\epsilon$-scalars for which we can assume $M_{\epsilon} \rightarrow \infty$ at the end of the calculation. Practical details about the implementation of $\epsilon$-scalars, in particular their Feynman rules, have been given in Refs. 5961.

Concerning renormalization, we use on-shell conditions for the bottom quark mass, one of the sbottom masses, and the sbottom quark mixing angle. The other sbottom mass is then fixed by $\mathrm{SU}(2)$ symmetry. The gluino does not require renormalization at this order. This top/stop-like renormalization scheme may cause perturbative problems for large $\mu_{\text {SUSY }}$ and $\tan \beta$ [62. However, due to our limit of degenerate sbottom masses we will restrict our analysis to moderate values of $\tan \beta$ anyway. The comparison of various other renormalization schemes is beyond the scope of the current paper and will be deferred to a forthcoming publication.

The external gluon wave functions $G_{\mu}$ are renormalized on-shell using

$$
\begin{aligned}
G_{\mu}^{\mathrm{B}} & =Z_{3}^{1 / 2} G_{\mu}, \\
Z_{3} & =1-\frac{\alpha_{s}^{\overline{\mathrm{DR}}}}{\pi} \frac{1}{\epsilon}\left(\frac{T}{6}\left(n_{s}+2\right)+\frac{C_{A}}{6}\right)+\delta z, \\
\delta z & =\frac{\alpha_{s}^{\overline{\mathrm{DR}}}}{\pi}\left(\frac{T}{12} \sum_{\substack{q \in\{b, t\} \\
i=1,2}} \ln \frac{\mu_{\mathrm{r}}^{2}}{\tilde{M}_{q i}^{2}}+\frac{T}{3} \ln \frac{\mu_{\mathrm{r}}^{2}}{M_{t}^{2}}+\frac{C_{A}}{6} \ln \frac{\mu_{\mathrm{r}}^{2}}{\tilde{M}_{g}^{2}}\right)-\frac{\alpha_{s}}{\pi} \frac{C_{A}}{6},
\end{aligned}
$$

\footnotetext{
${ }^{2}$ The factor of two can be deduced from the analytic structure of the amplitude.
} 
where $n_{s}=6$ is the number of squark flavors, $C_{A}=3$ and $T=1 / 2$ are color factors, and the last term in $\delta z$ is due to the $\epsilon$-scalars. In fact, at this order of $\alpha_{s}$, all of the finite part of $Z_{3}$ cancels against the conversion (see, e.g. Ref. [63])

$$
\alpha_{s}^{\overline{\mathrm{DR}}}=(1-\delta z) \alpha_{s}^{\overline{\mathrm{MS}},(5)},
$$

where $\alpha_{s}^{\overline{\mathrm{DR}}}$ denotes the strong coupling constant renormalized in the full MSSM according to $\overline{\mathrm{DR}}$ subtraction.

The bottom mass $M_{b}$, the sbottom mass $\tilde{M}_{b 1}$, and the sbottom mixing angle $\theta_{b}$ are renormalized on-shell in analogy to the top quark sector, see, e.g. Ref. [32]. The sbottom mass $\tilde{M}_{b 2}$ is determined from the SU(2) relation from the other (top and bottom) on-shell quark and squark masses and mixing angles, and its on-shell value $\tilde{M}_{b 2}^{\mathrm{OS}}$. The two mass definitions are related by a finite shift (see, e.g. Ref. [64] $\left.]^{3}\right)$ :

$$
\tilde{M}_{b 2}=\tilde{M}_{b 2}^{\mathrm{OS}}\left(1+\frac{\alpha_{s}}{\pi} \Delta_{\mathrm{OS}}^{b,(1)}\right) .
$$

The sbottom sector contribution to the Higgs production cross section can now be written as $\left(s_{n b}=\sin \left(n \theta_{b}\right), c_{n b}=\cos \left(n \theta_{b}\right)\right)$

$$
\begin{aligned}
\tilde{a}_{b}^{(1)}= & -\frac{M_{b}^{2}}{\tilde{M}_{b}^{2}} \frac{\sin \alpha}{\cos \beta}\left(\mathcal{K}_{1}^{b}-\frac{1}{2} \Delta_{\mathrm{OS}}^{b,(1)}\right)-\frac{\mu_{\mathrm{SUSY}}}{\tilde{M}_{b}} \frac{\cos (\alpha-\beta)}{\cos ^{2} \beta}\left(\mathcal{K}_{2}^{b}+\frac{1}{4} \sqrt{\frac{\tau_{q}}{\tilde{\tau}_{q}}} s_{2 b} \Delta_{\mathrm{OS}}^{b,(1)}\right) \\
& +\frac{M_{Z}^{2}}{\tilde{M}_{b}^{2}} \sin (\alpha+\beta)\left[\mathcal{K}_{3}^{b}-\frac{1}{4}\left(s_{b}^{2}+\frac{2}{3} c_{2 b} \sin ^{2} \theta_{W}\right) \Delta_{\mathrm{OS}}^{b,(1)}\right]+\mathcal{O}\left(\tilde{M}_{b}^{-4}\right),
\end{aligned}
$$

with the weak mixing angle $\theta_{W}$, cf. Eq. (34). Our result for the coefficients $\mathcal{K}_{i}^{q}$ is (cf. Eq. (1) )

$$
\begin{aligned}
& \mathcal{K}_{1}^{q}=\frac{25}{24}+\frac{17}{12} l_{q s}-\frac{5}{24}\left(B_{0}^{\mathrm{fin}}\left(\tau_{q}\right)-l_{\text {rq }}\right)+\frac{\tau_{q}}{2}+\tau_{q}\left(\frac{17}{24}-\frac{\tau_{q}}{2}\right) f\left(\tau_{q}\right), \\
& \mathcal{K}_{2}^{q}=-\frac{\tau_{q}}{2}\left[1+\left(1-\tau_{q}\right) f\left(\tau_{q}\right)\right]+\frac{\tau_{q}}{36 \tilde{\tau}_{q}}\left[-37+\frac{15}{2} l_{q s}-3 \tau_{q}-3\left(2-\tau_{q}-\tau_{q}^{2}\right) f\left(\tau_{q}\right)\right], \\
& \mathcal{K}_{3}^{q}=\frac{19}{32}+\frac{\tau_{q}}{6}+\frac{\tau_{q}}{6}\left(1-\tau_{q}\right) f\left(\tau_{q}\right) .
\end{aligned}
$$

Note that the $\mathcal{K}_{i}^{q}$ are given for a general quark flavor $q$, so we will be able to apply them also for the top contribution further below. The only difference arises from the functions

\footnotetext{
${ }^{3}$ Ref. 64 fixes $\tilde{M}_{b 2}$ on-shell and evaluates $\tilde{M}_{b 1}$ from the SU(2) condition; we found better numerical stability by fixing $\tilde{M}_{b 1}$ instead.
} 
$f(\tau)$, defined in Eq. (7), and $B_{0}^{\text {fin }}(\tau)$, given by

$$
B_{0}^{\text {fin }}\left(\tau_{q}\right)-l_{r q}= \begin{cases}2-2 \sqrt{\tau_{q}-1} \arctan \left(\frac{1}{\sqrt{\tau_{q}-1}}\right), & \tau_{q}>1, \\ 2-\sqrt{1-\tau_{q}}\left(\log \frac{1+\sqrt{1-\tau_{q}}}{1-\sqrt{1-\tau_{q}}}-i \pi\right), & \tau_{q} \leq 1 .\end{cases}
$$

Due to our limit of a degenerate SUSY mass spectrum, all the dependence on the squark mixing angle $\theta_{q}$ drops out in Eq. (17). This also removes all terms $\sim \tilde{M}_{g} / M_{q}$ found in Ref. [38]. In fact, many of the terms evaluated in that reference vanish in our approximation, while many of the terms of Eq. (17) vanish in the approximation of Ref. [38] since they are of higher orders in $M_{b}$ or $1 / \tilde{M}_{b}$. Needless to say that for the terms that are non-zero in both approximations we find complete agreement 4

Since the bottom quark mass is very small, the following expansion in $M_{b} / M_{h}$, which leads to particularly simple expressions, approximates Eq. (17) at the order of $1 \%$ for a reasonable range of masses [46]:

$$
\begin{aligned}
\mathcal{K}_{1}^{b} & =\frac{5}{8}+\frac{17}{12} l_{b s}+\frac{5}{24} L_{h b}+\mathcal{O}\left(\tau_{b}\right) \\
\mathcal{K}_{2}^{b} & =-\frac{\tau_{b}}{2}\left(1-\frac{L_{h b}^{2}}{4}\right)+\frac{\tau_{b}}{\tilde{\tau}_{b}}\left(-\frac{37}{36}+\frac{5}{24} l_{b s}+\frac{L_{h b}^{2}}{24}\right)+\mathcal{O}\left(\tau_{b}^{2}\right), \\
\mathcal{K}_{3}^{b} & =\frac{19}{32}+\frac{\tau_{b}}{6}\left(1-\frac{L_{h b}^{2}}{4}\right)+\mathcal{O}\left(\tau_{b}^{2}\right) .
\end{aligned}
$$

A particularly useful check of Eq. (17) is to evaluate it for $q=t$ and expand it in the limit $M_{t} \gg M_{h}$ :

$$
\begin{gathered}
\tilde{a}_{t}^{(1)}=\frac{M_{t}^{2}}{\tilde{M}_{t}^{2}} \frac{\cos \alpha}{\sin \beta} \mathcal{K}_{1}^{t}+\frac{\mu_{\mathrm{SUSY}}}{\tilde{M}_{t}} \frac{\cos (\alpha-\beta)}{\sin ^{2} \beta} \mathcal{K}_{2}^{t}-\frac{M_{Z}^{2}}{\tilde{M}_{t}^{2}} \sin (\alpha+\beta) \mathcal{K}_{3}^{t}+\mathcal{O}\left(\tilde{M}_{t}^{-4}\right) \\
\mathcal{K}_{1}^{t}=\frac{19}{12}+\frac{17}{12} l_{t s}+\frac{1}{120 \tau_{t}}+\mathcal{O}\left(1 / \tau_{t}^{2}\right) \\
\mathcal{K}_{2}^{t}=-\frac{1}{3}-\frac{7}{90 \tau_{t}}+\frac{\tau_{t}}{\tilde{\tau}_{t}}\left[-\frac{11}{12}+\frac{5}{24} l_{t s}+\mathcal{O}\left(1 / \tau_{t}\right)\right]+\mathcal{O}\left(1 / \tau_{t}^{2}\right), \\
\mathcal{K}_{3}^{t}=\frac{203}{288}+\frac{7}{270 \tau_{t}}+\mathcal{O}\left(1 / \tau_{t}^{2}\right) .
\end{gathered}
$$

This result can also be directly obtained by asymptotic expansions of the corresponding Feynman diagrams along the lines of Refs. [31 34]. Of course, we find complete agreement,

\footnotetext{
${ }^{4}$ Thanks to P. Slavich for confirmation.
} 
therefore validating the result of Eq. (17). Let us stress that we do not use Eq. (21) in our numerical analysis, but rather the more general results obtained with the help of evalcsusy.f [32].

\section{Total inclusive cross section}

\subsection{Scenarios}

In this section we present numerical results for the hadronic cross section for Higgs production in gluon fusion in the MSSM. Through $\mathcal{O}\left(\alpha_{s}^{3}\right)$, we include the effects from quark and squark loops as described above, i.e., the full quark mass dependence is kept for all leading order $\left(a_{q}^{(0)}\right)$ and real radiation contributions $\left(\Delta \sigma_{i j}\right)$, as well as for the virtual quark loop terms $\left(a_{q}^{(1)}\right)$. The virtual top squark and top/stop/gluino contribution is taken into account in the limit $\left\{M_{t}, \tilde{M}_{t 1}, \tilde{M}_{t 2}, \tilde{M}_{g}\right\} \gg M_{h}$, without further restrictions on the masses or SUSY parameters, with the help of evalcsusy.f. For the 2-loop virtual bottom squark and bottom/sbottom/gluino contribution, and only there, we apply the limit $\tilde{M}_{b 1}=\tilde{M}_{b 2}=\tilde{M}_{g} \equiv \tilde{M}_{b} \gg M_{h}, M_{b}$, where in numerical evaluations we set $\tilde{M}_{b} \equiv\left(\tilde{M}_{b 1}+\tilde{M}_{b 2}+\tilde{M}_{g}\right) / 3$. These approximations should be well-justified for the most popular SUSY benchmark scenarios (see, e.g. Refs. [30,65]), in particular since they are only applied to the virtual effects where the partonic center-of-mass energy is fixed to $\hat{s} \equiv M_{h}^{2}$.

In order to obtain numerical results for the cross section, one needs to insert numbers for the unknown SUSY parameters. In this paper, we will consider the following SUSY scenarios [30,66]:

$\underline{\text { gluophobic }( \pm) \text { : }}$

$$
\begin{aligned}
& M_{\mathrm{SUSY}}=350 \mathrm{GeV}, \quad \mu_{\mathrm{SUSY}}= \pm 300 \mathrm{GeV}, \\
& M_{2}=300 \mathrm{GeV}, \quad M_{3}=500 \mathrm{GeV}, \quad X_{t}=-750 \mathrm{GeV} \\
& \stackrel{\tan \beta=10}{\Rightarrow} \quad \tilde{M}_{t 1} \approx 150 \mathrm{GeV}, \quad \tilde{M}_{t 2} \approx 520 \mathrm{GeV}, \\
& \tilde{M}_{b 1} \approx 340 \mathrm{GeV}, \quad \tilde{M}_{b 2} \approx 370 \mathrm{GeV}, \quad \tilde{M}_{g}=500 \mathrm{GeV}
\end{aligned}
$$


$\underline{m_{h}^{\max }( \pm):}$

$$
\begin{array}{ll} 
& M_{\text {SUSY }}=1 \mathrm{TeV}, \quad \mu_{\mathrm{SUSY}}= \pm 200 \mathrm{GeV}, \\
& M_{2}=200 \mathrm{GeV}, \quad M_{3}=800 \mathrm{GeV}, \quad X_{t}=2 \mathrm{TeV} \\
\stackrel{\tan \beta=10}{\Rightarrow} \quad \tilde{M}_{t 1} \approx 830 \mathrm{GeV}, \quad \tilde{M}_{t 2} \approx 1170 \mathrm{GeV}, \\
& \tilde{M}_{b 1} \approx \tilde{M}_{b 2} \approx 1 \mathrm{TeV}, \quad \tilde{M}_{g}=800 \mathrm{GeV}
\end{array}
$$

$\underline{\operatorname{no}-m i x i n g}( \pm)$ :

$$
\begin{array}{cl} 
& M_{\mathrm{SUSY}}=2 \mathrm{TeV}, \quad \mu_{\mathrm{SUSY}}= \pm 200 \mathrm{GeV}, \\
& M_{2}=200 \mathrm{GeV}, \quad M_{3}=1600 \mathrm{GeV}, \quad X_{t}=0 \\
\stackrel{\tan \beta=10}{\Rightarrow} \quad \tilde{M}_{t 1} \approx \tilde{M}_{t 2} \approx \tilde{M}_{b 1} \approx \tilde{M}_{b 2} \approx 2 \mathrm{TeV}, \quad \tilde{M}_{g}=1600 \mathrm{GeV}
\end{array}
$$

$\underline{\text { small } \alpha_{\text {eff }}( \pm):}$

$$
\begin{array}{lll} 
& M_{\text {SUSY }}=800 \mathrm{GeV}, \quad \mu_{\mathrm{SUSY}}= \pm 2 \mathrm{TeV}, \\
& M_{2}=500 \mathrm{GeV}, \quad M_{3}=500 \mathrm{GeV}, \quad X_{t}=-1.1 \mathrm{TeV} \\
\stackrel{\tan \beta=10}{\Rightarrow} & \tilde{M}_{t 1} \approx 690 \mathrm{GeV}, \quad \tilde{M}_{t 2} \approx 920 \mathrm{GeV}, & \\
& \tilde{M}_{b 1}=760 \mathrm{GeV}, \quad \tilde{M}_{b 2}=840 \mathrm{GeV}, \quad \tilde{M}_{g}=500 \mathrm{GeV}
\end{array}
$$

The input parameters are the squark mass scale $M_{\mathrm{SUSY}}$, the bilinear Higgs coupling $\mu_{\mathrm{SUSY}}$, the gaugino mass parameters $M_{2}$ and $M_{3}$, and the off-diagonal term in the stop mixing matrix $M_{t} X_{t}$. Furthermore, it is always assumed that $A_{b}=A_{t} \equiv X_{t}+\mu_{\mathrm{SUSY}} / \tan \beta$, where $A_{b}$ and $A_{t}$ are trilinear couplings of the SUSY potential. For a more detailed description of these input parameters, let us refer to the documentation and references of the program FeynHiggs [67-70] which we use to determine the corresponding Higgs, sbottom and stop masses and mixing angles 5 The numbers for the squark masses quoted above are obtained for our default setting $\tan \beta=10$. For the quark masses, we use the input values

$$
M_{t}=173.3 \mathrm{GeV}, \quad m_{b}\left(m_{b}\right)=4.2 \mathrm{GeV} \quad \Rightarrow \quad M_{b}=4.79 \mathrm{GeV},
$$

where $M_{t}$ and $M_{b}$ are on-shell masses, and $m_{b}$ is the $\overline{\mathrm{MS}}$ running mass.

In the main part of this paper, we will restrict ourselves to the gluophobic(+) and/or the $m_{h}^{\max }(+)$ scenario. The results for negative sign, including the other two scenarios, are deferred to Appendix [].

\footnotetext{
${ }^{5}$ Once the theoretical accuracy of the Higgs cross section increases further, the three-loop result for the Higgs mass has to be taken into account 43, 71, 72.
} 


\subsection{Numerical results}

The total inclusive Higgs production cross section within the MSSM through NLO QCD is shown for the gluophobic $(+)$ and the $m_{h}^{\max }(+)$ scenario in Fig. $4(\mathrm{a})$ and (b), respectively. The figures include results for the Tevatron $(p \bar{p} @ 1.96 \mathrm{TeV})$ as well as for the LHC at various energies, where $\tan \beta=10$. Here and in the following, the renormalization and factorization scales are set to $\mu \equiv \mu_{\mathrm{f}}=\mu_{\mathrm{r}}=M_{h} / 2$ [28], unless indicated otherwise. Also shown is the corresponding light Higgs mass $M_{h}$, see Fig. 4 (c) and (d). The results for the other scenarios can be found in Appendix C.

In the SM, where the cross section has been studied in great detail $[9,22,24,22,73,174$, it was found that the NNLO QCD corrections are essential in order to reduce the scale uncertainty to an acceptable level. In addition, several calculations of beyond-NNLO effects lead to the conclusion that the fixed-order NNLO result provides a fairly precise prediction of the inclusive rate.

Since we expect a similar behavior in the MSSM, we need to transfer the available information from the SM result to the MSSM case. We therefore define our best prediction of the total inclusive cross section as

$$
\sigma^{\mathrm{MSSM}}=\sigma_{\mathrm{NLO}}^{\mathrm{MSSM}}+\left(g_{t}^{h}\right)^{2}\left[\left(1+\delta_{\mathrm{EW}}\right) \sigma_{\mathrm{NNLO}}^{\mathrm{SM}, t}-\sigma_{\mathrm{NLO}}^{\mathrm{SM}, t}\right]
$$

where $\sigma_{\mathrm{NLO}}^{\mathrm{MSSM}}$ is our result for the total inclusive cross section though $\mathcal{O}\left(\alpha_{s}^{3}\right)$ within the MSSM as described in the preceding sections. We consistently evaluate it with NLO parton density functions (PDFs) 6 The quantity $\sigma_{(\mathrm{N}) \mathrm{NLO}}^{\mathrm{SM}, t}$ is the top-quark induced SM cross section evaluated at (N)NLO QCD (i.e., with (N)NLO PDFs), and $g_{t}^{h}=\cos \alpha / \sin \beta$. In addition, the electro-weak correction factor within the SM, $\delta_{\mathrm{EW}}[27$, is included by assuming complete factorization, as it was indicated to be a reasonable assumption [28].

In this paper we restrict most of our analysis to moderate and small $\tan \beta$. Therefore, we do not actually expect the resummation of the dominant $\tan \beta$ terms along the lines of Ref. [76, 77] to be important. Nevertheless, we implement it, not least as a useful check: upon expansion of the resummed expression in terms of $\alpha_{s}$, we recover the coefficient $\mathcal{K}_{2}^{b}$ (through $\mathcal{O}\left(1 / \tilde{M}_{b}^{0}\right)$ ) which gives the leading term in $\tan \beta$ at NLO. The numerical effect will be studied in more detail below. The renormalization and factorization scales are again set to $\mu_{\mathrm{f}}=\mu_{\mathrm{r}}=M_{h} / 2$.

We provide the numerical results for $\sigma^{\mathrm{MSSM}}$ as well as the individual contributions from Eq. (27) for the gluophobic $(+)$ and the $m_{h}^{\max }(+)$ scenario in Table 1 and 2, As expected, in the gluophobic scenario the cross section is typically much smaller than the SM value

\footnotetext{
${ }^{6}$ In this paper, we use the central set of MSTW2008 [75] throughout. Detailed studies of the PDF dependence will be considered in a forthcoming paper.
} 


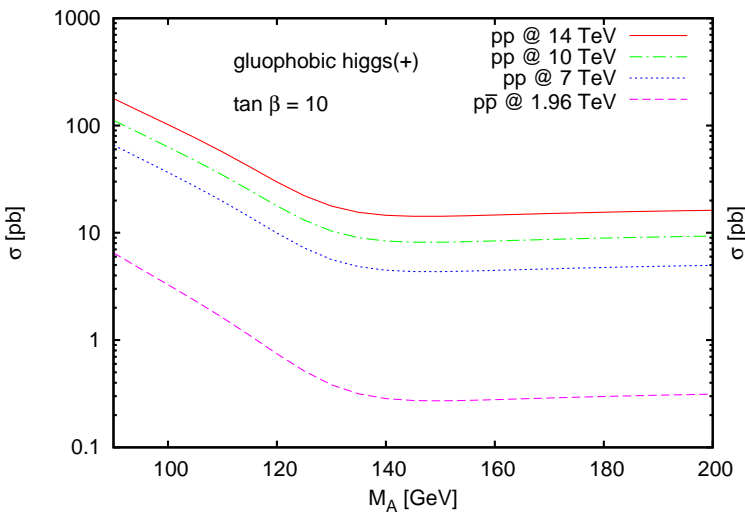

(a)

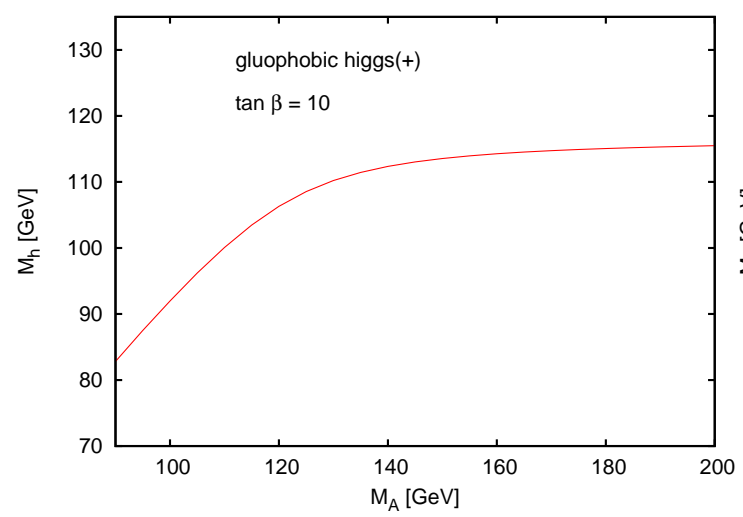

(c)

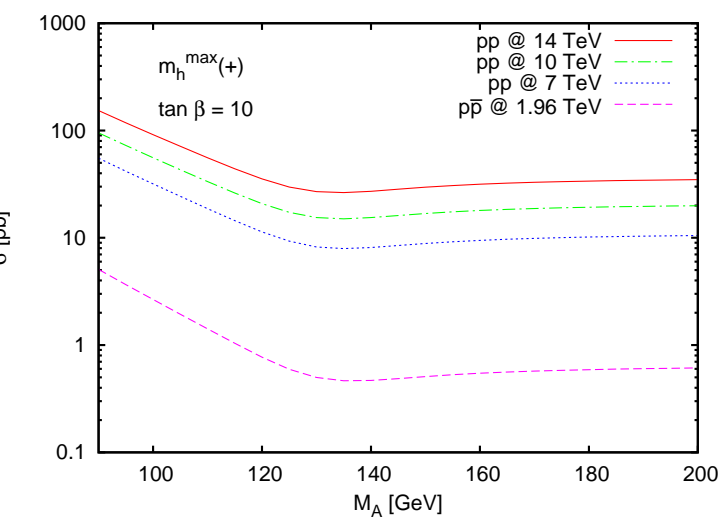

(b)

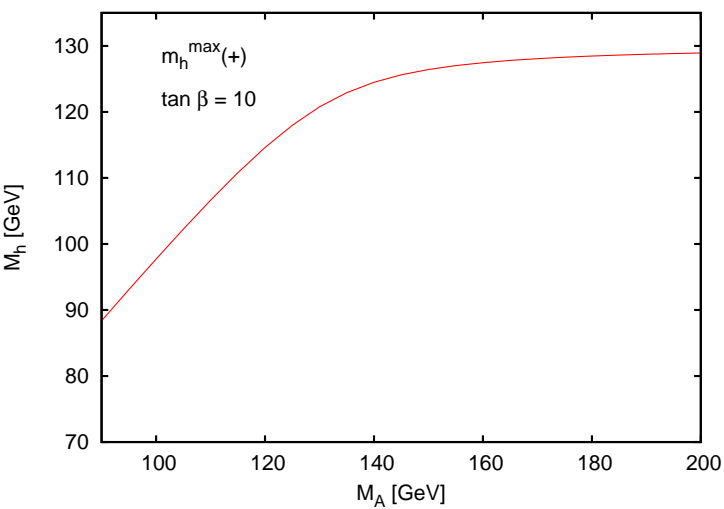

(d)

Figure 4: Inclusive total cross section for gluon fusion in the MSSM for the scenarios defined in Eqs. (22) and (23) at the Tevatron and the LHC for various energies. (a) gluophobic $(+)$; (b) $m_{h}^{\max }(+)$. Panels (c) and (d) show the corresponding light Higgs boson mass. 


\begin{tabular}{cccccccc}
$M_{A}$ & $M_{h}$ & $\left(g_{t}^{h}\right)^{2}$ & $1+\delta_{\mathrm{EW}}$ & $\sigma_{\mathrm{NLO}}^{\mathrm{SM}, t}$ & $\sigma_{\mathrm{NNLO}}^{\mathrm{SM}, t}$ & $\sigma_{\mathrm{NLO}}^{\mathrm{MSSM}}$ & $\sigma^{\mathrm{MSSM}}$ \\
\hline 90 & 82.8 & 0.063 & 1.037 & 31.49 & 37.28 & 65.90 & 66.35 \\
95 & 87.5 & 0.085 & 1.038 & 28.29 & 33.41 & 49.03 & 49.57 \\
100 & 92.0 & 0.119 & 1.039 & 25.63 & 30.20 & 36.53 & 37.21 \\
105 & 96.2 & 0.169 & 1.041 & 23.44 & 27.55 & 27.03 & 27.91 \\
110 & 100.1 & 0.240 & 1.042 & 21.65 & 25.40 & 19.69 & 20.85 \\
115 & 103.5 & 0.337 & 1.043 & 20.23 & 23.71 & 14.05 & 15.57 \\
120 & 106.3 & 0.452 & 1.044 & 19.14 & 22.41 & 9.93 & 11.86 \\
125 & 108.5 & 0.570 & 1.045 & 18.35 & 21.46 & 7.22 & 9.54 \\
130 & 110.2 & 0.672 & 1.046 & 17.78 & 20.78 & 5.64 & 8.30 \\
135 & 111.4 & 0.751 & 1.046 & 17.38 & 20.30 & 4.84 & 7.74 \\
140 & 112.4 & 0.809 & 1.046 & 17.09 & 19.96 & 4.48 & 7.55 \\
145 & 113.0 & 0.850 & 1.047 & 16.88 & 19.71 & 4.36 & 7.55 \\
150 & 113.5 & 0.880 & 1.047 & 16.72 & 19.52 & 4.35 & 7.62 \\
155 & 113.9 & 0.902 & 1.047 & 16.60 & 19.38 & 4.39 & 7.73 \\
160 & 114.3 & 0.919 & 1.047 & 16.50 & 19.26 & 4.46 & 7.84 \\
165 & 114.5 & 0.931 & 1.047 & 16.42 & 19.17 & 4.54 & 7.94 \\
170 & 114.7 & 0.941 & 1.047 & 16.36 & 19.09 & 4.61 & 8.04 \\
175 & 114.9 & 0.949 & 1.047 & 16.31 & 19.03 & 4.69 & 8.13 \\
180 & 115.1 & 0.955 & 1.047 & 16.26 & 18.98 & 4.75 & 8.21 \\
185 & 115.2 & 0.960 & 1.048 & 16.23 & 18.94 & 4.81 & 8.28 \\
190 & 115.3 & 0.964 & 1.048 & 16.19 & 18.90 & 4.87 & 8.35 \\
195 & 115.4 & 0.968 & 1.048 & 16.17 & 18.86 & 4.92 & 8.40 \\
200 & 115.5 & 0.971 & 1.048 & 16.14 & 18.84 & 4.97 & 8.46
\end{tabular}

Table 1: Data for the gluophobic(+) scenario with $\tan \beta=10$ at the LHC with $7 \mathrm{TeV}$. (Masses are given in $\mathrm{GeV}$, cross sections in pb.) 


\begin{tabular}{cccccccc}
$M_{A}$ & $M_{h}$ & $\left(g_{t}^{h}\right)^{2}$ & $1+\delta_{\mathrm{EW}}$ & $\sigma_{\mathrm{NLO}}^{\mathrm{SM}, t}$ & $\sigma_{\mathrm{NNLO}}^{\mathrm{SM}, t}$ & $\sigma_{\mathrm{NLO}}^{\mathrm{MSSM}}$ & $\sigma^{\mathrm{MSSM}}$ \\
\hline 90 & 88.4 & 0.030 & 1.038 & 27.74 & 32.74 & 55.20 & 55.38 \\
95 & 93.1 & 0.039 & 1.040 & 25.03 & 29.47 & 41.77 & 41.99 \\
100 & 97.7 & 0.054 & 1.041 & 22.72 & 26.68 & 31.82 & 32.09 \\
105 & 102.2 & 0.075 & 1.043 & 20.73 & 24.30 & 24.36 & 24.70 \\
110 & 106.6 & 0.106 & 1.044 & 19.03 & 22.28 & 18.72 & 19.17 \\
115 & 110.8 & 0.155 & 1.046 & 17.59 & 20.56 & 14.47 & 15.07 \\
120 & 114.6 & 0.227 & 1.047 & 16.40 & 19.14 & 11.36 & 12.19 \\
125 & 118.0 & 0.329 & 1.049 & 15.44 & 18.00 & 9.30 & 10.43 \\
130 & 120.8 & 0.453 & 1.050 & 14.70 & 17.13 & 8.22 & 9.70 \\
135 & 122.9 & 0.579 & 1.051 & 14.17 & 16.50 & 7.93 & 9.76 \\
140 & 124.5 & 0.687 & 1.051 & 13.80 & 16.06 & 8.11 & 10.22 \\
145 & 125.6 & 0.767 & 1.052 & 13.54 & 15.75 & 8.47 & 10.79 \\
150 & 126.4 & 0.824 & 1.052 & 13.36 & 15.54 & 8.85 & 11.31 \\
155 & 127.0 & 0.864 & 1.052 & 13.23 & 15.38 & 9.18 & 11.74 \\
160 & 127.5 & 0.892 & 1.052 & 13.13 & 15.27 & 9.46 & 12.08 \\
165 & 127.8 & 0.913 & 1.053 & 13.06 & 15.18 & 9.69 & 12.36 \\
170 & 128.1 & 0.928 & 1.053 & 13.00 & 15.11 & 9.88 & 12.58 \\
175 & 128.3 & 0.939 & 1.053 & 12.95 & 15.06 & 10.03 & 12.76 \\
180 & 128.5 & 0.948 & 1.053 & 12.91 & 15.01 & 10.16 & 12.90 \\
185 & 128.6 & 0.955 & 1.053 & 12.88 & 14.98 & 10.27 & 13.03 \\
190 & 128.7 & 0.961 & 1.053 & 12.86 & 14.94 & 10.36 & 13.13 \\
195 & 128.8 & 0.965 & 1.053 & 12.83 & 14.92 & 10.44 & 13.22 \\
200 & 128.9 & 0.969 & 1.053 & 12.81 & 14.90 & 10.51 & 13.29
\end{tabular}

Table 2: Data for the $m_{h}^{\max }(+)$ scenario with $\tan \beta=10$ at the LHC with $7 \mathrm{TeV}$. (Masses are given in $\mathrm{GeV}$, cross sections in pb.) 


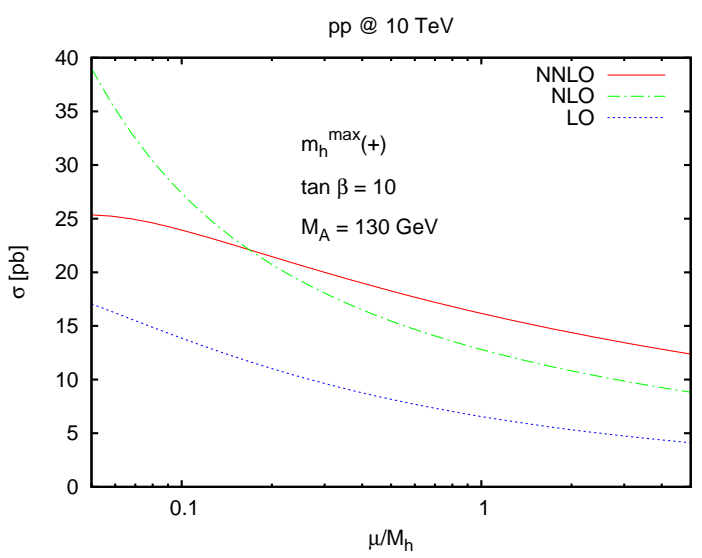

(a)

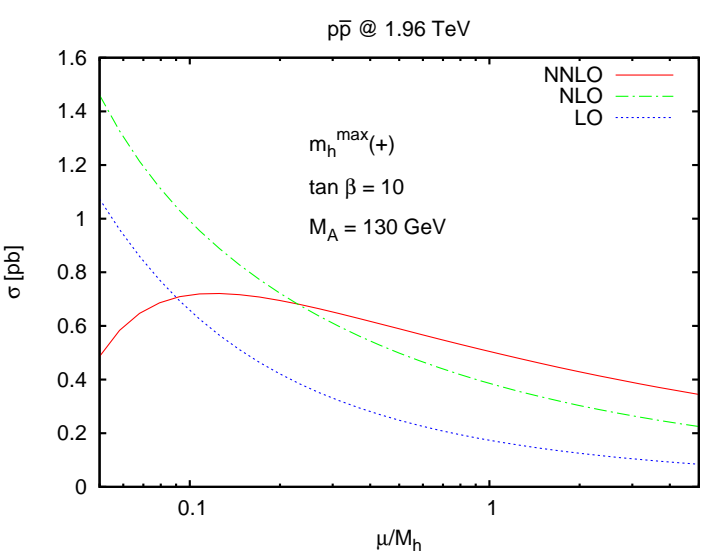

(b)

Figure 5: Renormalization/factorization scale dependence $\left(\mu=\mu_{\mathrm{f}}=\mu_{\mathrm{r}}\right)$ of the inclusive total cross section for gluon fusion in the MSSM. (a) LHC at $10 \mathrm{TeV}$; (b) Tevatron.

for the same Higgs mass, at least for Higgs masses above $\sim 100 \mathrm{GeV}$. In particular for the phenomenologically most relevant region $M_{h} \gtrsim 114 \mathrm{GeV}$, the ratio of the NLO MSSM to SM result is only 25-30\%. Inclusion of the top quark induced NNLO terms almost doubles the MSSM result, so that the MSSM to SM ratio increases to roughly 40-45\%. For $M_{h}<100 \mathrm{GeV}$, on the other hand, the MSSM cross section can become significantly larger than the SM result.

This qualitative feature of a suppression of the cross section due to SUSY effects for $M_{h} \gtrsim 114 \mathrm{GeV}$ applies also for the $m_{h}^{\max }$ scenario, but much less pronounced. And, also here, as one lowers the Higgs mass, the MSSM result surpasses the SM one.

Fig. 5 shows the scale variation of the MSSM cross section in the $m_{h}^{\max }(+)$ scenario at LO and NLO, as well as for our best prediction $\sigma^{\mathrm{MSSM}}$ (labelled "NNLO"). Renormalization and factorization scale are identified in this plot $\left(\mu=\mu_{\mathrm{f}}=\mu_{\mathrm{r}}\right)$, and varied by a factor 10 around $M_{h} / 2$. The lower order results exhibit a similar behavior as known from the SM, and, as expected, the inclusion of the NNLO top quark terms leads to a considerable stabilization against the scale variation. The error due to scale variations estimated from this plot by considering the variation within $M_{h} / 4 \leq \mu \leq M_{h}$ [28] is of the order of $15 \%$.

Fig. [6 shows the dependence of the cross section (Tevatron, $m_{h}^{\max }(+)$ ) on the choice of $\tan \beta$, where various contributions are displayed seperately. For example, the curve denoted $b+\tilde{b}$ is obtained by setting the top- and stop-Higgs couplings to zero, $g_{t}^{h}=\tilde{g}_{t, i j}^{h}=0$, 


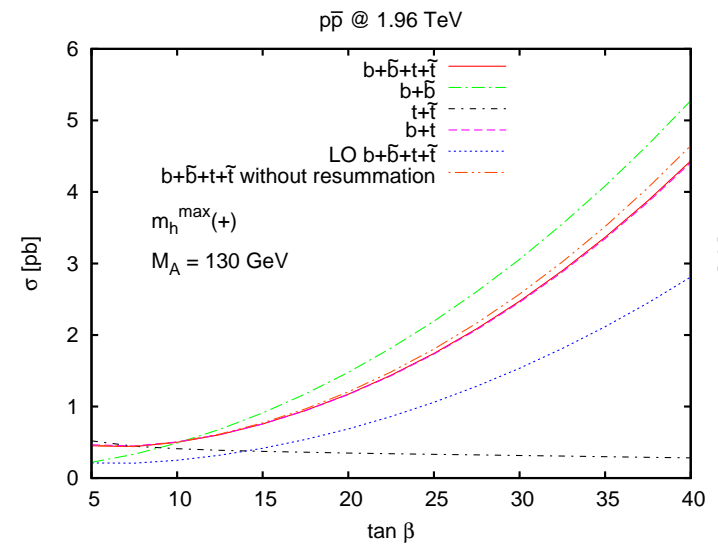

(a)

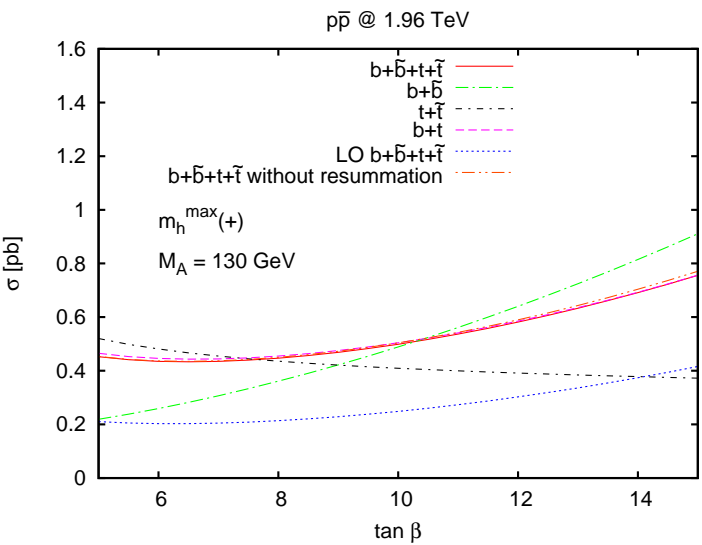

(b)

Figure 6: Dependence of (various contributions to) the cross section on $\tan \beta$ for Tevatron conditions. Panel (b) is an zoom of the low- to intermediate-tan $\beta$ region of panel (a).

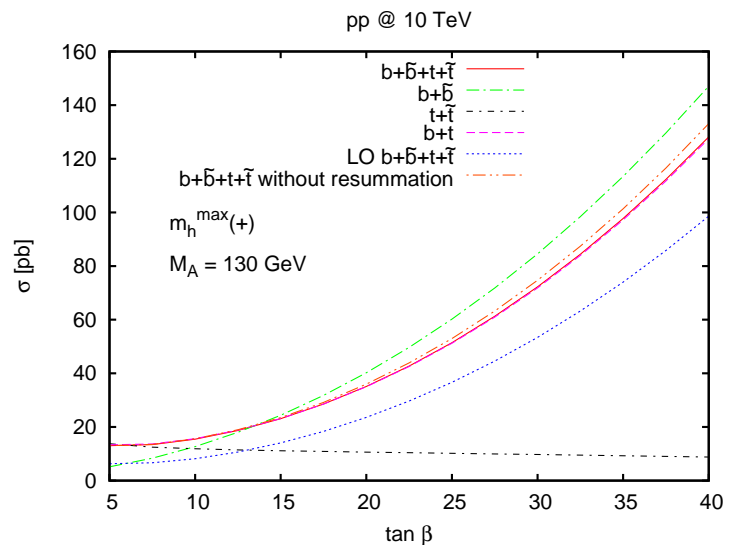

(a)

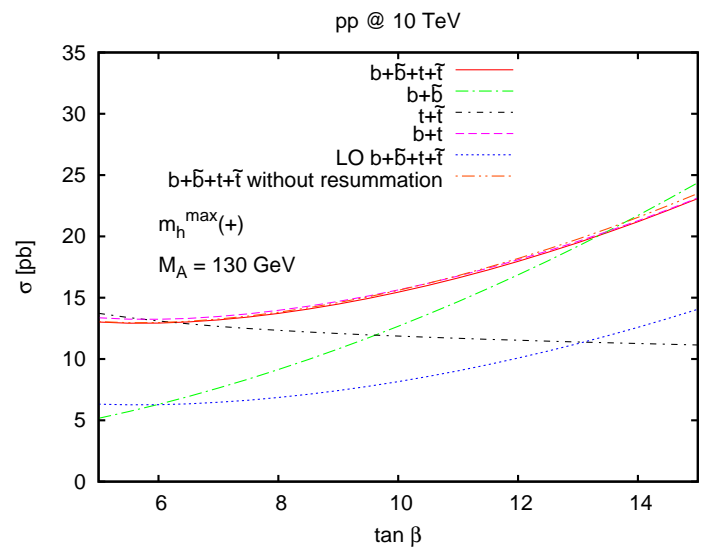

(b)

Figure 7: Same as Fig.6, but for the LHC at $10 \mathrm{TeV}$. 
etc. The figure illustrates that already for $\tan \beta \approx 10$, the bottom effects outweigh the top effects (green/long vs. black/short dash-dotted). Furthermore, squark effects have a significant effect even at these large squark masses of $\mathcal{O}(1 \mathrm{TeV})$ if the dominant $\tan \beta$ terms are not resummed in the Higgs-bottom Yukawa coupling (orange/dash-double-dotted vs. purple/dashed). Once this resummation is taken into account, the squark effects become negligible, however (purple/dashed vs. red/solid). Note that this plot depends strongly on the choice of $M_{A}$, of course. Another well-known feature that is shown is the effect of the NLO corrections which are of the order of $100 \%$ (blue/dotted vs. red/solid). A very similar behavior is observed for LHC conditions, see Fig. 7.

\section{Differential distributions}

In our perturbative partonic approach, the Higgs boson can only have non-zero transverse momentum $p_{T}$ when at least one parton is produced in association. Therefore, the purely virtual corrections do not contribute to the $p_{T}$ distribution $\mathrm{d} \sigma / \mathrm{d} p_{T}$. In fact, the LO $p_{T}$ distribution in the MSSM has been considered before [47, 49, 78, 79], and we include it here only for the sake of completeness. In Fig.8(a), the $p_{T}$ distribution at the LHC with $10 \mathrm{TeV}$ is displayed for both the SM and the MSSM in the $m_{h}^{\max }(+)$ scenario 7 Fig. 8 (b) diplays the ratio of the two curves. We observe that the shape of the $p_{T}$ distribution depends non-trivially on the model. The corresponding results for the Tevatron are shown in Fig.9, The kink at $p_{T} \approx 150 \mathrm{GeV}$ which is more pronounced in the SM, originates predominantly from the kinematical cut at $\sqrt{\hat{s}}=\sqrt{p_{T}^{2}+M_{h}^{2}}+p_{T}$ (see also Ref. [53]).

Concerning the rapidity distribution $\mathrm{d} \sigma / \mathrm{d} y$, where

$$
y \equiv \frac{1}{2} \ln \frac{E+p_{z}}{E-p_{z}},
$$

with $E$ and $p_{z}$ the energy and the longitudinal momentum of the Higgs boson in the lab frame, there clearly is a non-trivial distribution already at LO, given by

$$
\left.\frac{\mathrm{d} \sigma}{\mathrm{d} y}\right|_{\mathrm{LO}} \sim g\left(\sqrt{\tau} e^{y}\right) g\left(\sqrt{\tau} e^{-y}\right), \quad \tau=\frac{M_{h}^{2}}{s},
$$

where $s$ is the hadronic center-of-mass energy. Therefore, at NLO, also the virtual corrections need to be taken into account. We present here the first truly NLO results for this quantity in the MSSM. Fig.10(a) shows the rapidity distribution at the LHC with $10 \mathrm{TeV}$ both for the SM and the MSSM in the $m_{h}^{\max }(+)$ scenario, while Fig.10(b) shows again the ratio of the two curves. The model dependence of the shape is much smaller for the $y$ -

\footnotetext{
${ }^{7}$ Only the value of $M_{h}$ influences the SM prediction when changing the SUSY parameters.
} 


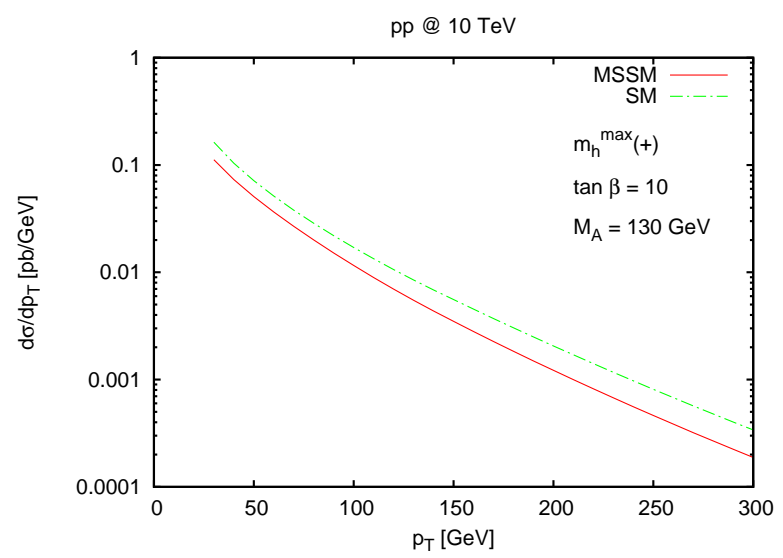

(a)

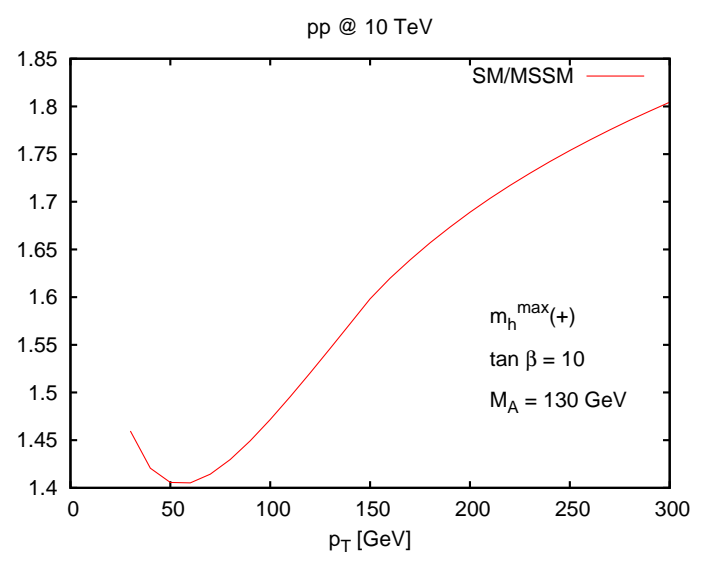

(b)

Figure 8: (a) Transverse momentum distribution of the Higgs boson at LO in the SM and the MSSM, using the $m_{h}^{\max }(+)$ scenario at the LHC for $10 \mathrm{TeV}$. (b) Ratio of the SM and the MSSM distribution.

than for the $p_{T}$-distribution. This is due to the fact that in the former case, a difference can arise only at NLO.

The same conclusions hold for the Tevatron, for which the results are displayed in Fig.11.

\section{$5 \quad$ Fourth matter generation}

It is well-known that a SM-like $4^{\text {th }}$ generation of quarks (denoted $\left(t_{4}, b_{4}\right)$ in what follows) would increase the Higgs production cross section significantly, leading to a much larger exclusion region from the Tevatron search results [8]. On the other hand, the Higgs exclusion from electro-weak precision data would soften considerably with a $4^{\text {th }}$ generation 80 . Effects of a $4^{\text {th }}$ generation on the inclusive $\mathrm{SM}_{4}$ Higgs cross section have been evaluated through NNLO QCD [81,82. At LO, also $H+$ jet and $H+2$ jet production has been studied in this model [82].

In this section, we evaluate the effect of an MSSM-like $4^{\text {th }}$ generation on the Higgs production cross section through NLO. Of course, also the SUSY constraints on the Higgs mass change significantly with the presence of a $4^{\text {th }}$ generation 83 85]. For this first study, we 


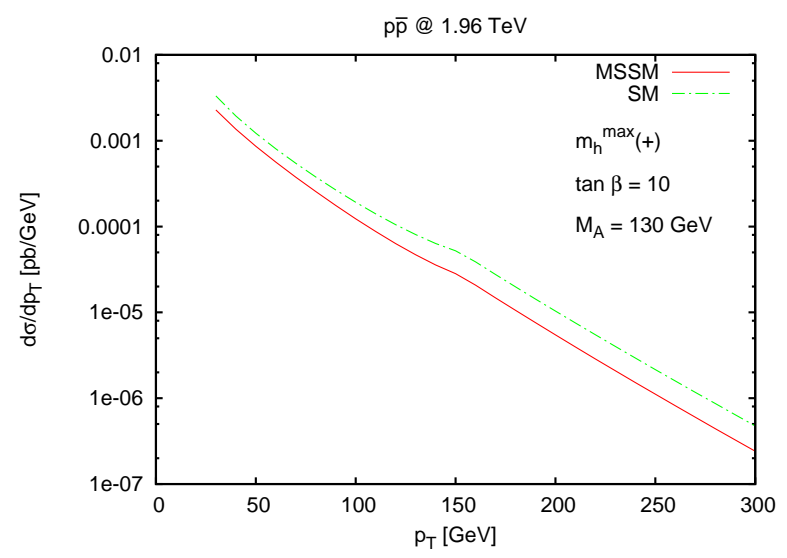

(a)

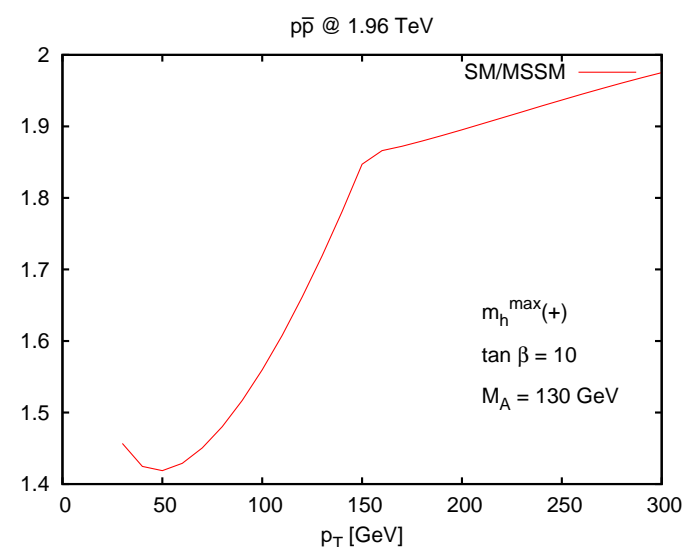

(b)

Figure 9: (a) Transverse momentum distribution of the Higgs boson at LO in the SM and the MSSM, using the $m_{h}^{\max }(+)$ scenario at the Tevatron. (b) Ratio of the SM and the MSSM distribution.

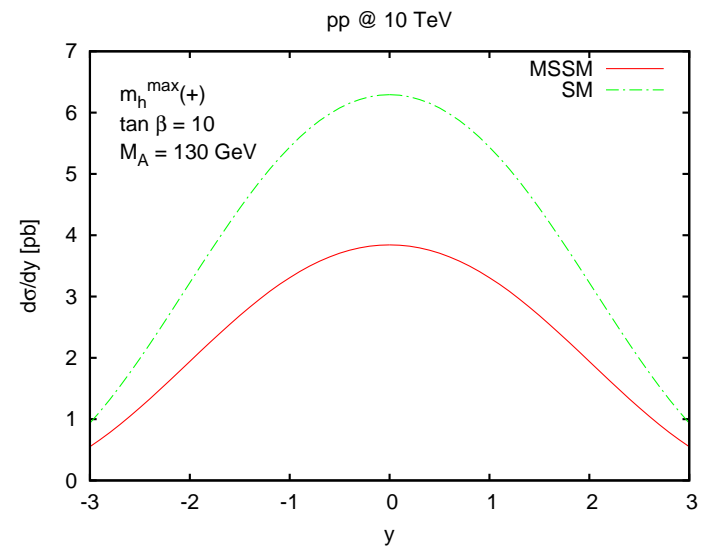

(a)

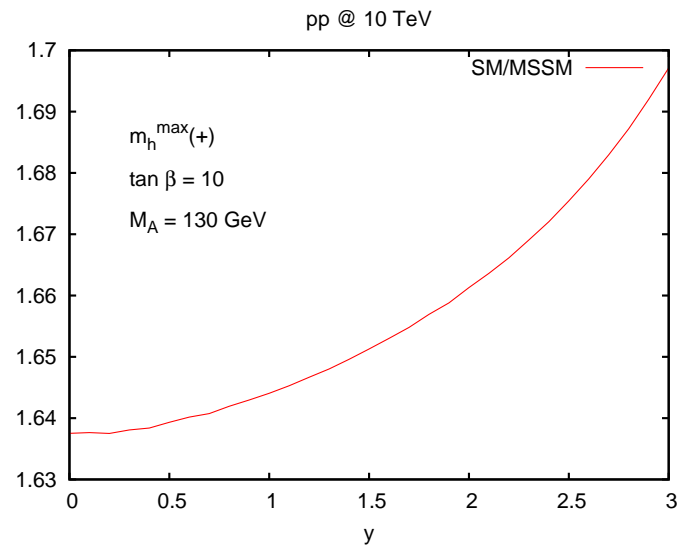

(b)

Figure 10: (a) Rapidity distribution of the Higgs boson at NLO in the SM and the MSSM, using the $m_{h}^{\max }(+)$ scenario at the LHC for $10 \mathrm{TeV}$. (b) Ratio of the MSSM and the SM distribution. 


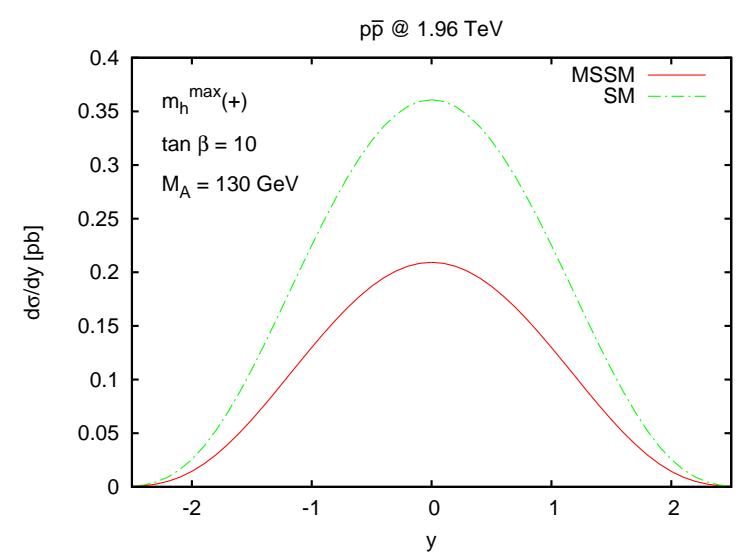

(a)

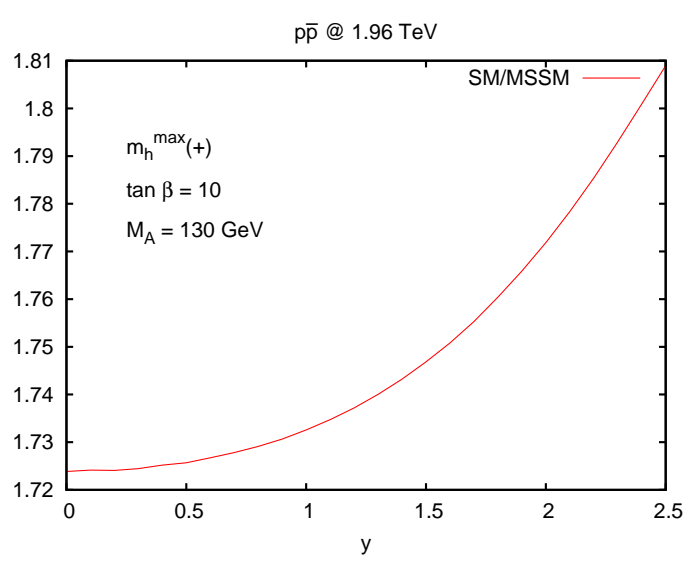

(b)

Figure 11: (a) Rapidity distribution of the Higgs boson at NLO in the SM and the MSSM, using the $m_{h}^{\max }(+)$ scenario at the Tevatron. (b) Ratio of the MSSM and the SM distribution.

will use the approximation

$$
M_{h}^{2}=\left(M_{h}^{\mathrm{MSSM}}\right)^{2}+\sum_{q=t_{4}, b_{4}} \frac{3}{2 \pi^{2}} \frac{M_{4}^{4}}{v^{2}} \log \frac{M_{S}^{2}}{M_{4}^{2}}, \quad M_{S}^{2}=M_{\mathrm{SUSY}}^{2}+M_{4}^{2},
$$

where $v=246 \mathrm{GeV}, M_{\mathrm{SUSY}}$ is given by the scenarios as defined in Sect. 3.1, and $M_{4}$ is the mass of the fourth generation quarks which are taken to be degenerate. The 3-generation result $M_{h}^{\mathrm{MSSM}}$ is again taken from FeynHiggs [67 70]. Furthermore, for the two lighter $4^{\text {th }}$ generation squark masses we also assume $\tilde{M}_{q 1}=M_{4}\left(q \in\left\{b_{4}, t_{4}\right\}\right)$, while the heavier ones are set to $\tilde{M}_{q 2}=\left(2 M_{\text {SUSY }}^{2}+M_{4}^{2}\right)^{1 / 2}$. The form of the coupling constants for the $4^{\text {th }}$ generation (s)quarks is taken to be identical to the first three generations, i.e., they are given by Eqs. (33) and (37) of Appendix A, with the obvious replacements of the masses and mixing angles. For recent $4^{\text {th }}$ generation search limits, see Refs. [86, 87].

Fig.12(a) displays the Tevatron and LHC results for the $\mathrm{SM}_{4}$ and the $\mathrm{MSSM}_{4}$ using the SUSY parameters as in the $m_{h}^{\max }(+)$ scenario. In order to avoid non-perturbativity of the $h b_{4} \bar{b}_{4}$ coupling, we set $\tan \beta=1$. On top of the well-known factor of $\sim 9$ from the $\mathrm{SM} \rightarrow \mathrm{SM}_{4}$ transition, we find a $30 \%$ decrease when switching to the $\mathrm{MSSM}_{4}$. This value depends quite sensitively on the actual values of the $4^{\text {th }}$ generation squark masses and suggests further, more detailed investigations. 


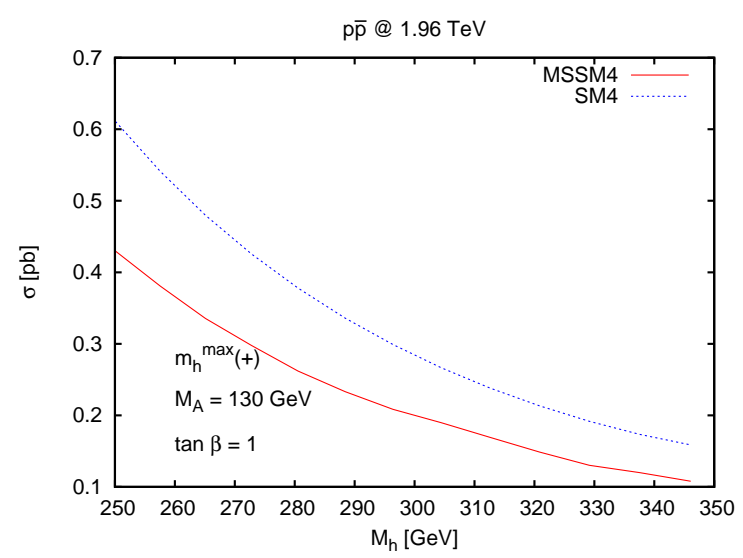

(a)

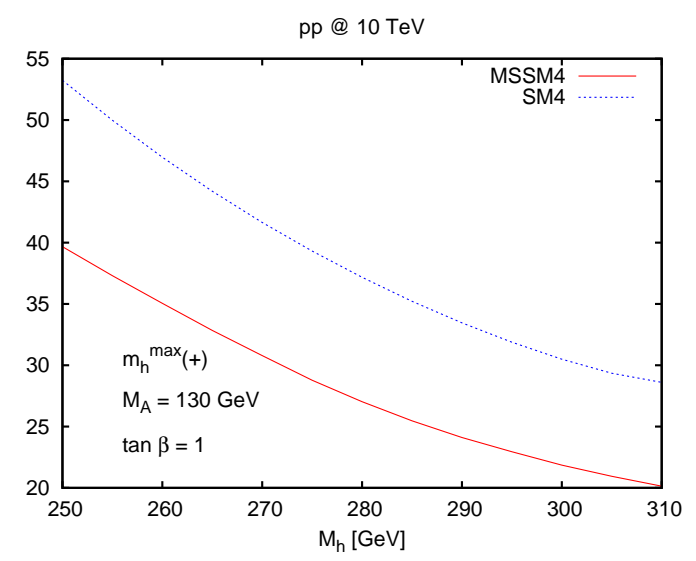

(b)

Figure 12: Higgs production cross section as a function of the light Higgs boson mass (a) at the Tevatron and (b) at the LHC, with a $4^{\text {th }}$ SM- and MSSM-like generation of (s)fermions.

\section{Conclusions}

The various contributions of the NLO QCD corrections to the cross section for Higgs production in gluon fusion have been combined in order to evaluate it consistently within the MSSM. Both quark and squark effects have been taken into account in the top and the bottom sector, including the mixed quark/squark/gluino and all interference effects. The numerical results have been presented for a set of selected MSSM scenarios, but our implementation allows for any other reasonable set of parameters.

For the total inclusive cross section, we constructed a "best approximation" which includes the known NNLO QCD top-quark induced corrections. We also provided results for Higgs distributions in $p_{T}$ through LO and in $y$ through NLO. Finally, the effect of a $4^{\text {th }}$ MSSM generation on the Higgs production rate has been investigated.

In future works, we will study the effect of changing the renormalization scheme, in particular for the bottom/sbottom sector, include the recently evaluated NNLO effects for the top/stop sector, and consider also the production of the heavy Higgs (see also Ref. [38]).

We hope that these results, plus similar ones provided on-line [88, will be useful input for experimental analyses. We will gladly provide numbers for other scenarios to the interested reader, and are planning to release the code for public use in the near future. 
Acknowledgments. We would like to thank G. Degrassi, S. Heinemeyer, K. Ozeren, P. Slavich, and G. Weiglein for enlightening comments and discussions. Furthermore, we thank M. Steinhauser and N. Zerf for pointing out a number of typos in the original version of the manuscript. This work was supported by DFG, contract HA 2990/3-1.

\section{A Feynman rules and coupling constants}

The Feynman rules for the SUSY-QCD vertices can be found in Refs. [32, 61]. Here, we give in addition the ones for the bottom- and sbottom-Higgs vertices:
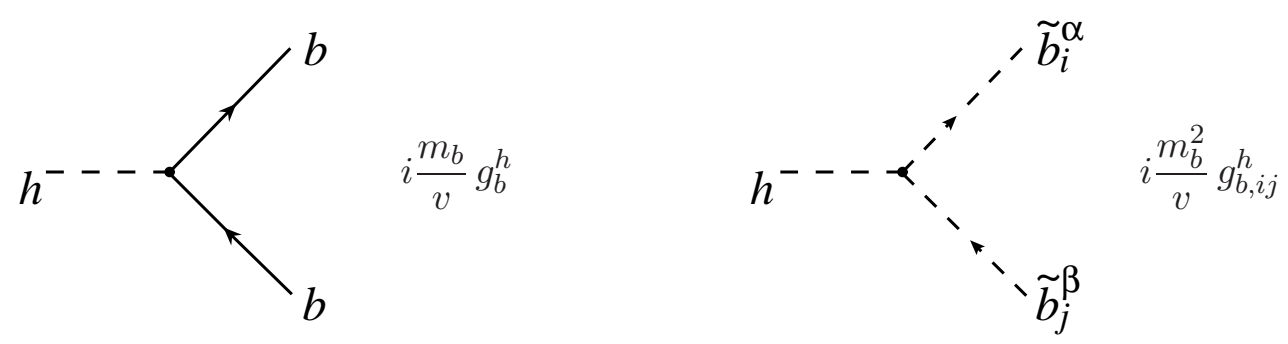

The bottom-Higgs coupling constant reads

$$
g_{b}^{h}=-\frac{\sin \alpha}{\cos \beta},
$$

and the sbottom-Higgs couplings are

$$
g_{b, i j}^{h}=g_{b, i j}^{h, \mathrm{EW}}+g_{b, i j}^{h, \mu}+g_{b, i j}^{h, \alpha},
$$


with

$$
\begin{aligned}
g_{b, 11}^{h, \mathrm{EW}} & =c_{1, b}^{\mathrm{EW}} \cos ^{2} \theta_{b}+c_{2, b}^{\mathrm{EW}} \sin ^{2} \theta_{b}, \\
g_{b, 22}^{h, \mathrm{EW}} & =c_{1, b}^{\mathrm{EW}} \sin ^{2} \theta_{b}+c_{2, b}^{\mathrm{EW}} \cos ^{2} \theta_{b}, \\
g_{b, 12}^{h, \mathrm{EW}} & =g_{b, 21}^{h, \mathrm{EW}}=\frac{1}{2}\left(c_{2, b}^{\mathrm{EW}}-c_{1, b}^{\mathrm{EW}}\right) \sin 2 \theta_{b}, \\
g_{b, 11}^{h, \mu} & =-g_{b, 22}^{h, \mu}=-\frac{\mu_{\mathrm{SUSY}}}{M_{b}} \frac{\cos (\alpha-\beta)}{\cos ^{2} \beta} \sin 2 \theta_{b}, \\
g_{b, 12}^{h, \mu} & =g_{b, 21}^{h, \mu}=-\frac{\mu_{\mathrm{SUSY}}}{M_{b}} \frac{\cos (\alpha-\beta)}{\cos ^{2} \beta} \cos 2 \theta_{b}, \\
g_{b, 11}^{h, \alpha} & =-\frac{\sin \alpha}{\cos \beta}\left[2+\frac{\tilde{M}_{b 1}^{2}-\tilde{M}_{b 2}^{2}}{2 M_{b}^{2}} \sin ^{2} 2 \theta_{b}\right], \\
g_{b, 22}^{h, \alpha} & =-\frac{\sin \alpha}{\cos \beta}\left[2-\frac{\tilde{M}_{b 1}^{2}-\tilde{M}_{b 2}^{2}}{2 M_{b}^{2}} \sin ^{2} 2 \theta_{b}\right], \\
g_{b, 12}^{h, \alpha} & =g_{b, 21}^{h, \alpha}=-\frac{\sin \alpha}{\cos \beta} \frac{\tilde{M}_{b 1}^{2}-\tilde{M}_{b 2}^{2}}{2 M_{b}^{2}} \sin 2 \theta_{b} \cos 2 \theta_{b},
\end{aligned}
$$

where

$$
\begin{aligned}
c_{1, b}^{\mathrm{EW}} & =\frac{2 M_{Z}^{2}}{M_{q}^{2}}\left(\left|I_{3}\right|-|Q| \sin ^{2} \theta_{W}\right) \sin (\alpha+\beta), \\
c_{2, b}^{\mathrm{EW}} & =\frac{2 M_{Z}^{2}}{M_{q}^{2}}|Q| \sin ^{2} \theta_{W} \sin (\alpha+\beta), \\
\sin \theta_{W} & =\sqrt{1-\frac{M_{W}^{2}}{M_{Z}^{2}}}, \quad I_{3}=-\frac{1}{2}, \quad Q=-\frac{1}{3},
\end{aligned}
$$

and

$$
\tan \beta=\frac{v_{2}}{v_{1}}, \quad v=\frac{2 M_{W}}{g}=\frac{1}{\sqrt{\sqrt{2} G_{F}}}=\sqrt{v_{1}^{2}+v_{2}^{2}} \approx 246 \mathrm{GeV}
$$

with $v_{1}, v_{2}$ the vacuum expectation values of the two Higgs doublets. The angle $\theta_{b}$ rotates the super-partners of the left- and right-handed bottom quarks into their mass eigenstates, while $\alpha$ does the same for the neutral components of the Higgs doublets. In Eq. (33) we have already expressed the trilinear couplings of the soft SUSY breaking terms through independent parameters:

$$
A_{b}=\frac{\tilde{M}_{b 1}^{2}-\tilde{M}_{b 2}^{2}}{2 M_{b}} \sin 2 \theta_{b}+\mu_{\mathrm{SUSY}} \tan \beta .
$$


The expressions in Eq. (33) and (34) are completely analogous to the ones for the scalar top sector, given in Ref. 32]. They can be transformed into each other by the replacements

$$
\begin{aligned}
t \leftrightarrow b, \quad & \mu_{\mathrm{SUSY}} \leftrightarrow-\mu_{\mathrm{SUSY}}, \quad \alpha \leftrightarrow \alpha+\frac{\pi}{2}, \quad \beta \leftrightarrow \beta+\frac{\pi}{2}, \\
& Q \leftrightarrow Q-1, \quad I_{3} \leftrightarrow I_{3}-1 .
\end{aligned}
$$

\section{B Expansion of quark terms}

Analytic expressions for the virtual quark loop contribution to the gluon fusion process at NLO have been presented in 8 Refs $[35,36,44$. It may be convenient for the reader to

have the relevant limits of these expressions. We quote them here for the large quark mass limit,

$$
\begin{aligned}
\frac{a_{q}^{(1)}}{g_{q}^{h}}= & \frac{11}{4}+\frac{1237}{1080} \tau_{q}^{-1}+\frac{17863}{28350} \tau_{q}^{-2}+\frac{157483}{396900} \tau^{3}+\frac{636694}{2338875} \tau_{q}^{-4} \\
& +\frac{48712384706}{245827456875} \tau_{q}^{-5}+\frac{96272051048}{639151387875} \tau_{q}^{-6}+\frac{6428929236304}{54327867969375} \tau_{q}^{-7} \\
& +\frac{11720258014074752}{122835309478756875} \tau_{q}^{-8}+\frac{24899957625820672}{316945428161236875} \tau_{q}^{-9}+\mathcal{O}\left(\tau_{q}^{-10}\right)
\end{aligned}
$$

and for the small quark mass limit:

$$
\begin{aligned}
\frac{a_{q}^{(1)}}{g_{q}^{h}}= & \frac{\tau_{q}}{4}\left[47-31 \zeta_{3}-18 \zeta_{4}+L_{h q}\left(-12-4 \zeta_{2}+11 \zeta_{3}\right)+L_{h q}^{2}\left(-\frac{9}{4}-\frac{1}{2} \zeta_{2}\right)\right. \\
& \left.+L_{h q}^{3}-\frac{5}{48} L_{h q}^{4}\right]+\left(\frac{\tau_{q}}{4}\right)^{2}\left[1-10 \zeta_{2}+128 \zeta_{3}+72 \zeta_{4}+L_{h q}\left(-2-44 \zeta_{3}\right)\right. \\
& \left.+L_{h q}^{2}\left(-\frac{11}{2}+2 \zeta_{2}\right)-9 L_{h q}^{3}+\frac{5}{12} L_{h q}^{4}\right]+\left(\frac{\tau_{q}}{4}\right)^{3}\left[\frac{1073}{8}+\frac{109}{2} \zeta_{2}-80 \zeta_{3}\right. \\
& \left.+36 \zeta_{4}+L_{h q}\left(\frac{543}{4}+40 \zeta_{2}-32 \zeta_{3}\right)+L_{h q}^{2}\left(\frac{179}{2}+8 \zeta_{2}\right)+\frac{119}{12} L_{h q}^{3}+\frac{1}{6} L_{h q}^{4}\right] \\
& +\mathcal{O}\left(\tau_{q}^{4}\right),
\end{aligned}
$$

where $\zeta_{n} \equiv \zeta(n)$ is Riemann's zeta function evaluated at $n$, with

$$
\zeta_{2}=\frac{\pi^{2}}{6}=1.64493 \ldots, \quad \zeta_{3}=1.20206 \ldots, \quad \zeta_{4}=\frac{\pi^{4}}{90}=1.08232 \ldots .
$$

\footnotetext{
${ }^{8}$ For the sake of clarity, let us remark that in the notation of Ref. [44, it is $F_{0}^{H} B_{1}^{H}=2 a_{q}^{(1)} / g_{q}^{h}$.
} 


\section{Total cross section for other SUSY scenarios}

This section collects the results for the total inclusive cross section in the SUSY scenarios of Eqs. (22) - (25) which have not been discussed in the main text. We restrict ourselves to graphical representations; the corresponding data tables can be found at the URL [88. Figs.13 15 are simply the analogues of Fig. 4 for these scenarios.

\section{References}

[1] The TEVNPH Working Group of the CDF and D0 Collaborations, Combined CDF and DO Upper Limits on Standard Model Higgs-Boson Production with up to $6.7 \mathrm{fb}^{-1}$ of Data, arXiv: 1007.4587.

[2] A. Djouadi, The anatomy of electro-weak symmetry breaking. I: The Higgs boson in the standard model, Phys. Reports 457 (2008) 1, hep-ph/0503172.

[3] A. Djouadi, The anatomy of electro-weak symmetry breaking. II: The Higgs bosons in the minimal supersymmetric model, Phys. Reports 459 (2008) 1, hep-ph/0503173.

[4] R. Harlander, Higgs production - Higher orders and finite top mass effects, Proceedings of the XLVth Rencontres de Moriond 2010.

[5] R. Harlander, Higgs Production At The Large Hadron Collider: Theoretical Status, J. Phys. G 35 (2008) 033001.

[6] Tevatron New Phenomena \& Higgs Working Group, Combined CDF and D0 upper limits on MSSM Higgs boson production in $\tau \tau$ final states with up to 2.2 $\mathrm{fb}^{-1}$, arXiv:1003.3363.

[7] J. Baglio, A. Djouadi, Revisiting the constraints on the Supersymmetric Higgs sector at the Tevatron, arXiv:1012.2748.

[8] The CDF Collaboration and D0 Collaboration (T. Aaltonen et al.), Combined Tevatron upper limit on $g g \rightarrow H \rightarrow W^{+} W^{-}$and constraints on the Higgs boson mass in fourth-generation fermion models, arXiv:1005.3216.

[9] H.M. Georgi, S.L. Glashow, M.E. Machacek, D.V. Nanopoulos, Higgs Bosons From Two Gluon Annihilation In Proton Proton Collisions, Phys. Rev. Lett. 40 (1978) 692.

[10] A. Djouadi, M. Spira, P.M. Zerwas, Production of Higgs bosons in proton colliders: QCD corrections, Phys. Lett. B 264 (1991) 440. 


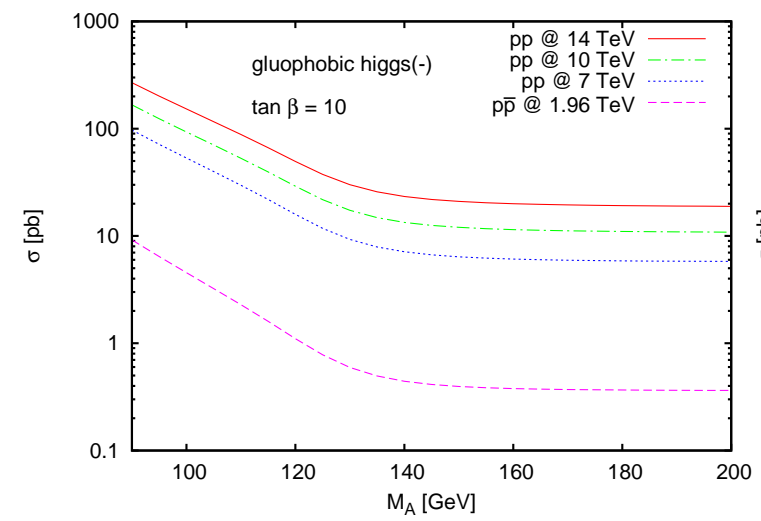

(a)

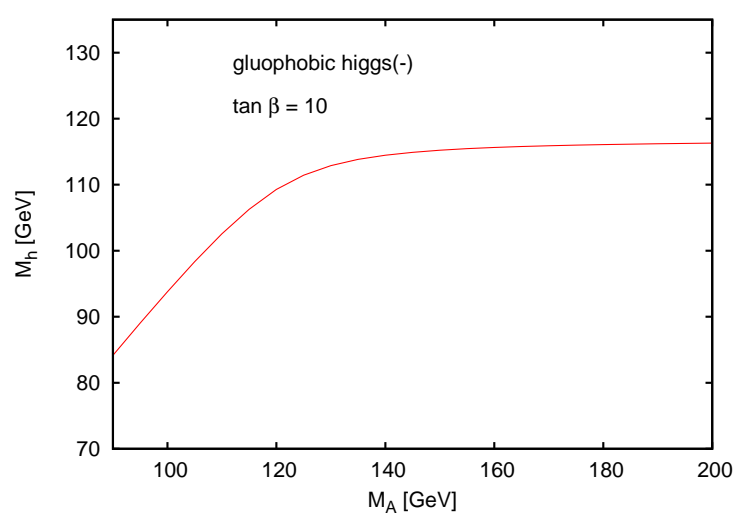

(c)

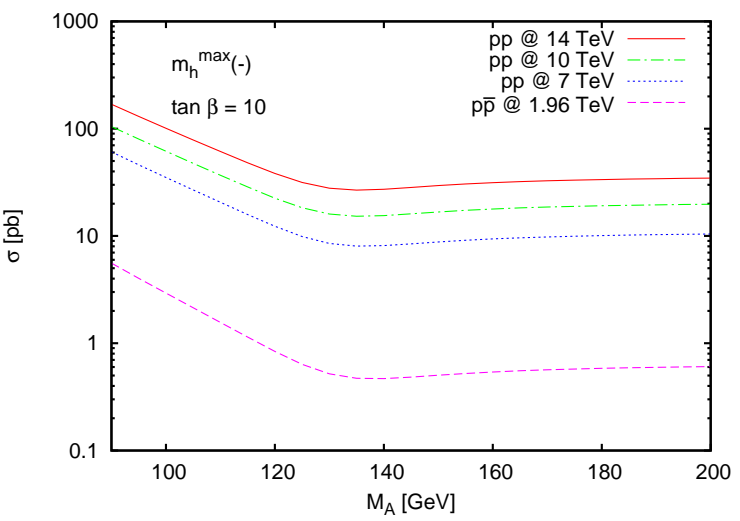

(b)

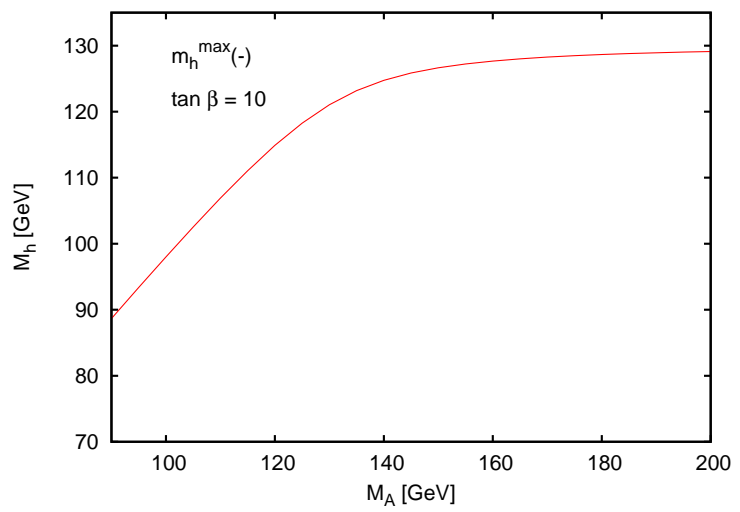

(d)

Figure 13: Inclusive total cross section for gluon fusion in the MSSM. (a) gluophobic(-); (b) $m_{h}^{\max }(-)$. Panels (c) and (d) show the corresponding light Higgs boson mass. Note that the scenarios gluophobic $(+)$ and $m_{h}^{\max }(+)$ can be found in Fig.4. 


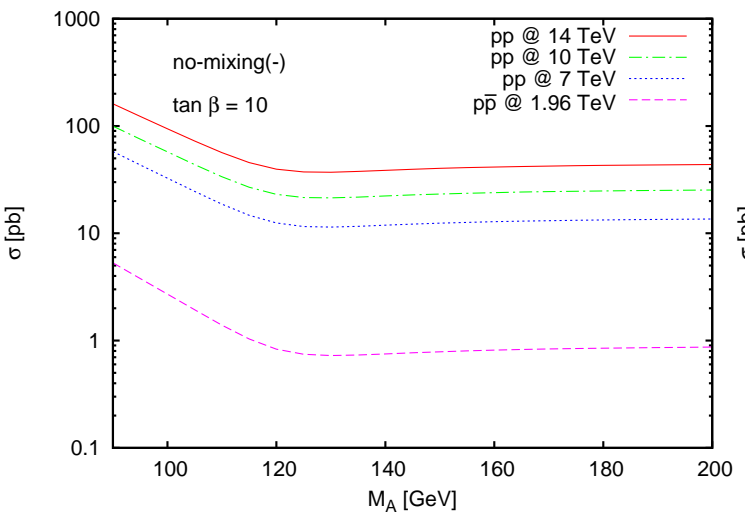

(a)

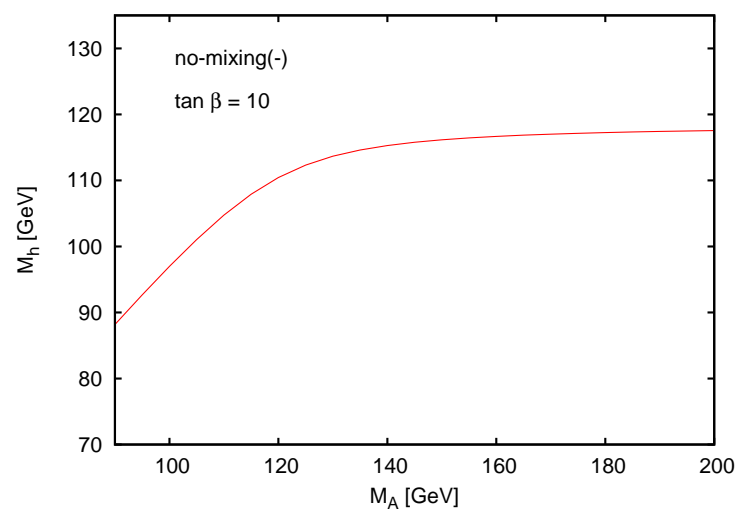

(c)

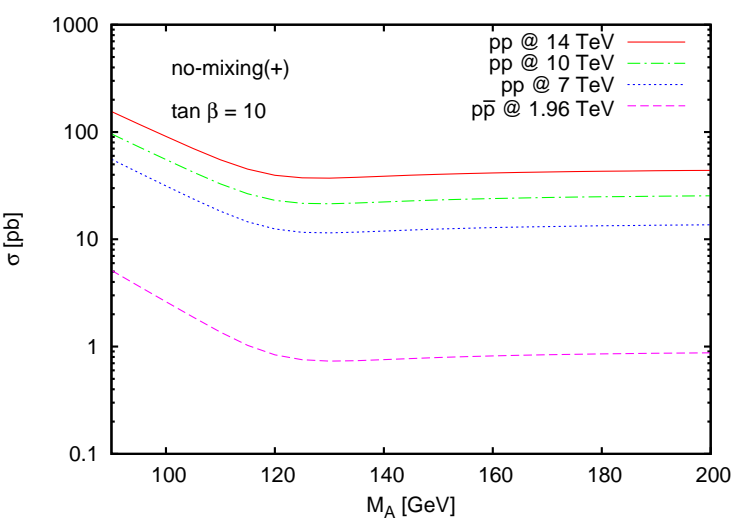

(b)

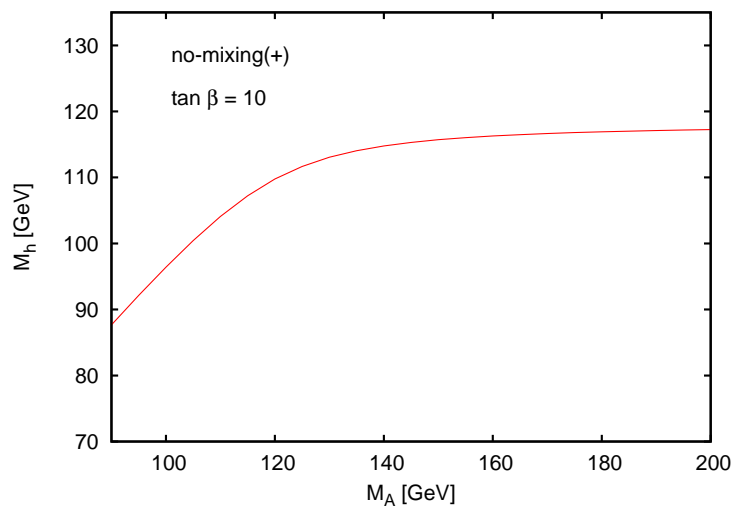

(d)

Figure 14: Inclusive total cross section for gluon fusion in the MSSM. (a) nomixing (-); (b) no-mixing(+). Panels (c) and (d) show the corresponding light Higgs boson mass. 


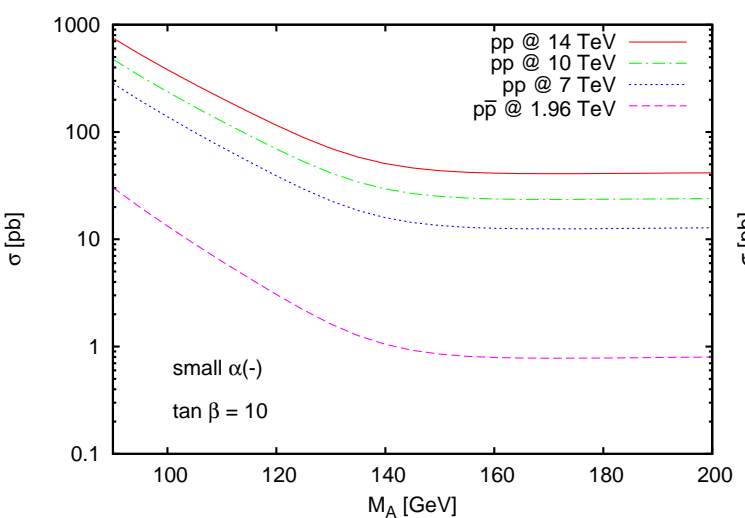

(a)

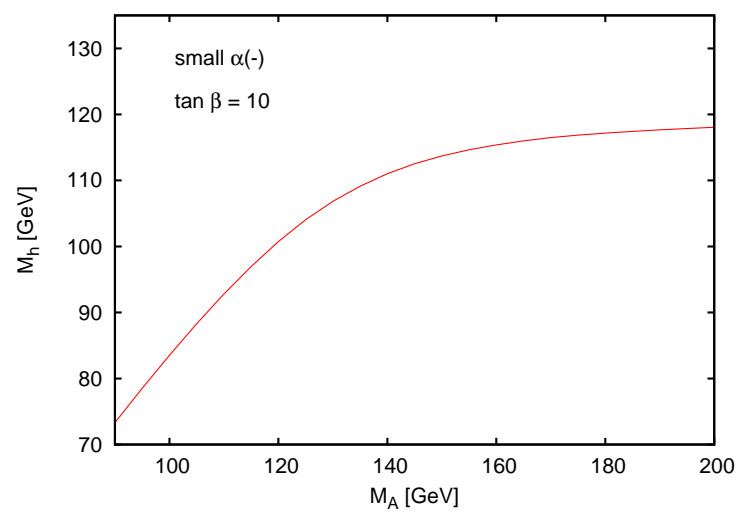

(c)

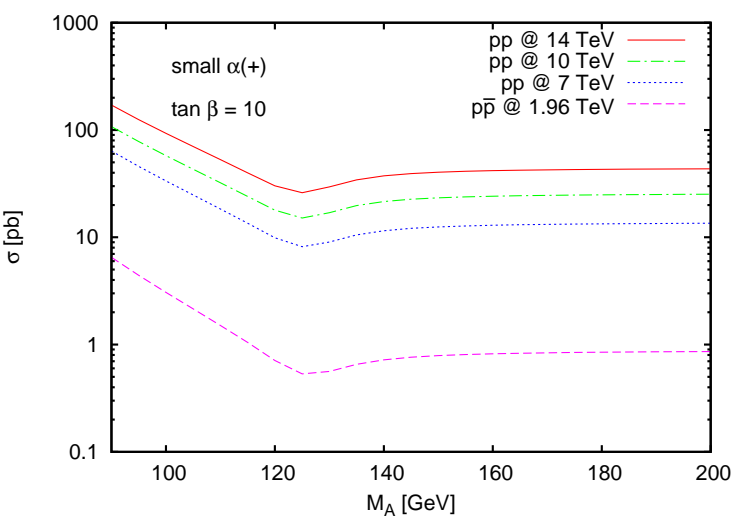

(b)

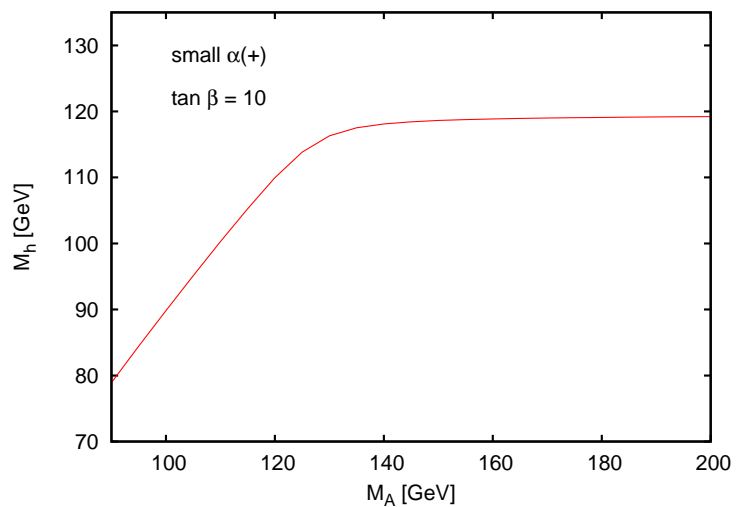

(d)

Figure 15: Inclusive total cross section for gluon fusion in the MSSM. (a) small$\alpha_{\text {eff }}(-)$; (b) small- $\alpha_{\text {eff }}(+)$. Panels (c) and (d) show the corresponding light Higgs boson mass. 
[11] S. Dawson, Radiative corrections to Higgs boson production, Nucl. Phys. B 359 (1991) 283.

[12] M. Spira, A. Djouadi, D. Graudenz, P.M. Zerwas, Higgs boson production at the LHC, Nucl. Phys. B 453 (1995) 17, hep-ph/9504378.

[13] R.V. Harlander and W.B. Kilgore, Next-to-next-to-leading order Higgs production at hadron colliders, Phys. Rev. Lett. 88 (2002) 201801, hep-ph/0201206.

[14] C. Anastasiou and K. Melnikov, Higgs boson production at hadron colliders in NNLO QCD, Nucl. Phys. B 646 (2002) 220, hep-ph/0207004.

[15] V. Ravindran, J. Smith, W.L. van Neerven, NNLO corrections to the total cross section for Higgs boson production in hadron hadron collisions, Nucl. Phys. B 665 (2003) 325, hep-ph/0302135.

[16] S. Marzani, R.D. Ball, V. Del Duca, S. Forte, A. Vicini, Higgs production via gluongluon fusion with finite top mass beyond next-to-leading order, Nucl. Phys. B 800 (2008) 127, arXiv:0801.2544.

[17] R.V. Harlander and K.J. Ozeren, Finite top mass effects for hadronic Higgs production at next-to-next-to-leading order, JHEP 0911 (2009) 088, arXiv:0909.3420.

[18] A. Pak, M. Rogal, M. Steinhauser, Finite top quark mass effects in NNLO Higgs boson production at LHC, JHEP 1002 (2010) 025, arXiv:0911.4662.

[19] R.V. Harlander, H. Mantler, S. Marzani, K.J. Ozeren, Higgs production in gluon fusion at next-to-next-to-leading order QCD for finite top mass, Eur. Phys. J. C 66 (2010) 359, arXiv:0912.2104.

[20] S. Catani, D. de Florian, M. Grazzini, P. Nason, Soft-gluon resummation for Higgs boson production at hadron colliders, JHEP 0307 (2003) 028, hep-ph/0306211.

[21] A.Idilbi, X.-d. Ji, J.P. Ma, F. Yuan, Threshold resummation for Higgs production in effective field theory, Phys. Rev. D 73 (2006) 077501, hep-ph/0509294.

[22] A. Idilbi, X.-d. Ji, F. Yuan, Resummation of threshold logarithms in effective field theory for DIS, Drell-Yan and Higgs production, Nucl. Phys. B 753 (2006) 42, hep-ph/0605068.

[23] V. Ravindran, Higher-order threshold effects to inclusive processes in QCD, Nucl. Phys. B 752 (2006) 173, hep-ph/0603041.

[24] S. Moch and A. Vogt, Higher-order soft corrections to lepton pair and Higgs boson production, Phys. Lett. B 631 (2005) 48, hep-ph/0508265. 
[25] V. Ahrens, T. Becher, M. Neubert, L.L. Yang, Renormalization-Group Improved Prediction for Higgs Production at Hadron Colliders, Eur. Phys. J. C 62 (2009) 333, arXiv:0809.4283.

[26] U. Aglietti, R. Bonciani, G. Degrassi, A. Vicini, Two-loop light fermion contribution to Higgs production and decays, Phys. Lett. B 595 (2004) 432, hep-ph/0404071.

[27] S. Actis, G. Passarino, C. Sturm and S. Uccirati, NLO Electroweak Corrections to Higgs Boson Production at Hadron Colliders, Phys. Lett. B 670 (2008) 12, arXiv:0809.1301.

[28] C. Anastasiou, R. Boughezal, F. Petriello, Mixed QCD-electroweak corrections to Higgs boson production in gluon fusion, JHEP 0904 (2009) 003, arXiv:0811.3458.

[29] A. Djouadi, Squark effects on Higgs boson production and decay at the LHC, Phys. Lett. B 435 (1998) 101, hep-ph/9806315.

[30] M. Carena, S. Heinemeyer, C. E. M. Wagner, G. Weiglein, Suggestions for benchmark scenarios for MSSM Higgs boson searches at hadron colliders, Eur. Phys. J. C 26 (2003) 601, hep-ph/0202167.

[31] R.V. Harlander and M. Steinhauser, Hadronic Higgs Production and Decay in Supersymmetry at Next-to-Leading Order, Phys. Lett. B 574 (2003) 258-268, hep-ph/0307346.

[32] R.V. Harlander and M. Steinhauser, Supersymmetric Higgs production in gluon fusion at next-to-leading order, JHEP 0409 (2004) 066, hep-ph/0409010.

[33] R.V. Harlander and M. Steinhauser, Effects of SUSY-QCD in hadronic Higgs production at next-to-next-to-leading order, Phys. Rev. D 68 (2003) 111701, hep-ph/0308210.

[34] G. Degrassi and P. Slavich, On the NLO QCD corrections to Higgs production and decay in the MSSM, Nucl. Phys. B 805 (2008) 267, arXiv:0806.1495.

[35] C. Anastasiou, S. Beerli, S. Bucherer, A. Daleo, Z. Kunszt, Two-loop amplitudes and master integrals for the production of a Higgs boson via a massive quark and a scalar-quark loop, JHEP 0701 (2007) 082, hep-ph/0611236.

[36] U. Aglietti, R. Bonciani, G. Degrassi, A. Vicini, Analytic results for virtual QCD corrections to Higgs production and decay, JHEP 0701 (2007) 021, hep-ph/0611266.

[37] M. Mühlleitner and M. Spira, Higgs boson production via gluon fusion: Squark loops at NLO QCD, Nucl. Phys. B 790 (2008) 1, hep-ph/0612254. 
[38] G. Degrassi and P. Slavich, NLO QCD bottom corrections to Higgs boson production in the MSSM, JHEP 1011 (2010) 044, arXiv:1007.3465.

[39] C. Anastasiou, S. Beerli and A. Daleo, The two-loop QCD amplitude gg-i $h, H$ in the Minimal Supersymmetric Standard Model, Phys. Rev. Lett. 100 (2008) 241806, arXiv:0803.3065.

[40] A. Pak, M. Steinhauser, N. Zerf, Higgs boson production in gluon fusion to NNLO in the MSSM, arXiv:1012.0639.

[41] S. Heinemeyer, MSSM Higgs physics at higher orders, Int. J. Mod. Phys. A 21 (2006) 2659 , hep-ph/0407244.

[42] B.C. Allanach, A. Djouadi, J.L. Kneur, W. Porod, P. Slavich, Precise determination of the neutral Higgs boson masses in the MSSM, JHEP 0409 (2004) 044, hep-ph/0406166.

[43] P. Kant, R.V. Harlander, L. Mihaila, M. Steinhauser, Light MSSM Higgs boson mass to three-loop accuracy, arXiv:1005.5709, JHEP 1008 (2010) 104.

[44] R. Harlander and P. Kant, Higgs production and decay: Analytic results at next-toleading order QCD, JHEP 0512 (2005) 015, hep-ph/0509189.

[45] The source code can be obtained from the URL http://www-ttp.physik. uni-karlsruhe.de/Progdata/ttp04/ttp04-19/

[46] F. Hofmann, Influence of the Supersymmetric Bottom Sector on Higgs Production and Decay, Dissertation at Wuppertal University, May 2009.

[47] O. Brein and W. Hollik, MSSM Higgs bosons associated with high-p $p_{T}$ jets at hadron colliders, Phys. Rev. D 68 (2003) 095006, hep-ph/0305321.

[48] B. Field, S. Dawson, J. Smith, Scalar and pseudoscalar Higgs boson plus one jet production at the LHC and Tevatron, Phys. Rev. D 69 (2004) 074013, hep-ph/0311199.

[49] O. Brein and W. Hollik, Distributions for MSSM Higgs boson + jet production at hadron colliders, Phys. Rev. D 76 (2007) 035002, arXiv:0705.2744.

[50] G. Passarino and M.J.G. Veltman, One loop corrections for $e^{+} e^{-}$annihilation into $\mu^{+} \mu^{-}$in the Weinberg model, Nucl. Phys. B 160 (1979) 151.

[51] R.K. Ellis, I. Hinchliffe, M. Soldate, J.J. van der Bij, Higgs Decay To $\tau^{+} \tau^{-}: A$ Possible Signature Of Intermediate Mass Higgs Bosons At The SSC, Nucl. Phys. B 297 (1988) 221. 
[52] U. Baur and E.W.N. Glover, Higgs Boson Production At Large Transverse Momentum In Hadronic Collisions, Nucl. Phys. B 339 (1990) 38.

[53] W.Y. Keung and F. Petriello, Electroweak and finite quark-mass effects on the Higgs boson transverse momentum distribution, Phys. Rev. D 80 (2009) 013007, arXiv:0905.2775.

[54] R. Bonciani, G. Degrassi and A. Vicini, Scalar Particle Contribution to Higgs Production via Gluon Fusion at NLO, JHEP 0711 (2007) 095, arXiv:0709.4227.

[55] R.V. Harlander and K.J. Ozeren, Top mass effects in Higgs production at nextto-next-to-leading order QCD: virtual corrections, Phys. Lett. B 679 (2009) 467, arXiv:0907.2997.

[56] A. Pak, M. Rogal, M. Steinhauser, Virtual three-loop corrections to Higgs boson production in gluon fusion for finite top quark mass, Phys. Lett. B 679 (2009) 473, arXiv:0907.2998.

[57] V.A. Smirnov, Applied asymptotic expansions in momenta and masses, Springer Tracts in Modern Physics, Vol. 177 (2002), ISBN 3-540-42334-6.

[58] M. Steinhauser, MATAD: A program package for the computation of massive tadpoles, Comp. Phys. Commun. 134 (2001) 335, hep-ph/0009029.

[59] R. Harlander, P. Kant, L. Mihaila, M. Steinhauser, Dimensional reduction applied to QCD at three loops, JHEP 09 (2006) 053, hep-ph/0607240.

[60] R.V. Harlander, D.R.T. Jones, P. Kant, L. Mihaila, M. Steinhauser, Four-loop beta function and mass anomalous dimension in Dimensional Reduction, JHEP 0612 (2006) 024, hep-ph/0610206.

[61] R.V. Harlander, L. Mihaila, M. Steinhauser, The SUSY-QCD beta function to three loops, JHEP 63 (2009) 383, arXiv:0905.4807.

[62] A. Brignole, G. Degrassi, P. Slavich, F. Zwirner, On the two loop sbottom corrections to the neutral Higgs boson masses in the MSSM, Nucl. Phys. B 643 (2002) 79, hep-ph/0206101.

[63] R. Harlander, L. Mihaila, M. Steinhauser, Two-loop matching coefficients for the strong coupling in the MSSM, Phys. Rev. D 72 (2005) 095009, hep-ph/0509048.

[64] S. Heinemeyer, W. Hollik, H. Rzehak, G. Weiglein, High-precision predictions for the MSSM Higgs sector at $\mathcal{O}\left(\alpha_{b} \alpha_{s}\right)$, Eur. Phys. J. C 39 (2005) 465, hep-ph/0411114. 
[65] B.C. Allanach et al., The Snowmass points and slopes: Benchmarks for SUSY searches, in Proc. of the APS/DPF/DPB Summer Study on the Future of Particle Physics (Snowmass 2001) ed. N. Graf, Eur. Phys. J. C 25 (2002) 113, [eConf C010630 (2001) P125], hep-ph/0202233.

[66] M.S. Carena, S. Heinemeyer, C.E.M. Wagner, G. Weiglein, MSSM Higgs boson searches at the Tevatron and the LHC: Impact of different benchmark scenarios, Eur. Phys. J. C 45 (2006) 797, hep-ph/0511023..

[67] M. Frank, T. Hahn, S. Heinemeyer, W. Hollik, H. Rzehak, G. Weiglein, The Higgs boson masses and mixings of the complex MSSM in the Feynman-diagrammatic approach, JHEP 0702 (2007) 047, hep-ph/0611326.

[68] G. Degrassi, S. Heinemeyer, W. Hollik, P. Slavich, G. Weiglein, Towards highprecision predictions for the MSSM Higgs sector, Eur. Phys. J. C 28 (2003) 133, hep-ph/0212020.

[69] S. Heinemeyer, W. Hollik, G. Weiglein, The masses of the neutral CP-even Higgs bosons in the MSSM: Accurate analysis at the two-loop level, Eur. Phys. J. C 9 (1999) 343, hep-ph/9812472.

[70] S. Heinemeyer, W. Hollik, G. Weiglein, FeynHiggs: A program for the calculation of the masses of the neutral CP-even Higgs bosons in the MSSM, Comp. Phys. Commun. 124 (2000) 76, hep-ph/9812320.

[71] S. P. Martin, Three-loop corrections to the lightest Higgs scalar boson mass in supersymmetry, Phys. Lett. B 75 (2007) 055005, hep-ph/0701051.

[72] R.V. Harlander, P. Kant, L. Mihaila, M. Steinhauser, Higgs boson mass in supersymmetry to three loops, Phys. Rev. Lett. 100 (2008) 191602; (E) ibid. 101 (2008) 039901, arXiv:0803.0672.

[73] D. de Florian and M. Grazzini, Higgs production through gluon fusion: updated cross sections at the Tevatron and the LHC, Phys. Lett. B 674 (2009) 291, arXiv:0901.2427.

[74] J. Baglio, A. Djouadi, Higgs production at the lHC, arXiv:1012.0530.

[75] A.D. Martin, W.J. Stirling, R.S. Thorne, G. Watt, Parton distributions for the LHC, Eur. Phys. J. C 63 (2009) 189, arXiv:0901.0002.

[76] M.S. Carena, D. Garcia, U. Nierste, C.E.M. Wagner, Effective Lagrangian for the $\bar{t} b H^{+}$interaction in the MSSM and charged Higgs phenomenology, Nucl. Phys. B 577 (2000) 88, hep-ph/9912516. 
[77] M. Carena, D. Garcia, U. Nierste and C.E.M. Wagner, $b \rightarrow s \gamma$ and supersymmetry with large $\tan \beta$, Phys. Lett. B 499 (2001) 141, hep-ph/0010003.

[78] M. Spira, HIGLU: A Program for the Calculation of the Total Higgs Production Cross Section at Hadron Colliders via Gluon Fusion including QCD Corrections, hep-ph/9510347.

[79] U. Langenegger, M. Spira, A. Starodumov, P. Trueb, SM and MSSM Higgs Boson Production: Spectra at large transverse Momentum, JHEP 0606 (2006) 035, hep-ph/0604156.

[80] G.D. Kribs, T. Plehn, M. Spannowsky, T.M.P. Tait, Four generations and Higgs physics, Phys. Rev. D 76 (2007) 075016, arXiv:0706.3718.

[81] C. Anastasiou, R. Boughezal, E. Furlan, The NNLO gluon fusion Higgs production cross-section with many heavy quarks, JHEP 1006 (2010) 101, arXiv:1003.4677.

[82] Q. Li, M. Spira, J. Gao, C.S. Li, Higgs Boson Production via Gluon Fusion in the Standard Model with four Generations, arXiv:1011.4484.

[83] R. Fok, G.D. Kribs, Four Generations, the Electroweak Phase Transition, and Supersymmetry, Phys. Rev. D 78 (2008) 075023, arXiv:0803.4207.

[84] S. Litsey, M. Sher, Higgs Masses in the Four Generation MSSM, Phys. Rev. D 80 (2009) 057701, arXiv:0908.0502.

[85] S. Dawson, P. Jaiswal, Four Generations, Higgs Physics, and the MSSM, Phys. Rev. D 82 (2010) 073017, arXiv:1009.1099.

[86] T. Aaltonen et al. [CDF Collaboration], Search for new bottomlike quark pair decays $Q \bar{Q} \rightarrow\left(t W^{\mp}\right)\left(\bar{t} W^{ \pm}\right)$in Same-Charge Dilepton Events, Phys. Rev. Lett. 104 (2010) 091801, arXiv:0912.1057.

[87] D. Cox [CDF Collaboration], Search for a heavy top $t^{\prime} \rightarrow W q$ in top events, arXiv:0910.3279.

[88] http://particle.uni-wuppertal.de/harlander/research/data/gghbsusy 